\title{
The First Fundamental Theorem of Coinvariant Theory for the Quantum General Linear Group
}

By

\author{
Kenneth R. GoOdearL*, Thomas H. Lenagan** and Laurent RigaL***
}

\begin{abstract}
We prove First Fundamental Theorems of Coinvariant Theory for the standard coactions of the quantum groups $\mathcal{O}_{q}\left(G L_{t}(K)\right)$ and $\mathcal{O}_{q}\left(S L_{t}(K)\right)$ on the quantized algebra $\mathcal{O}_{q}\left(M_{m, t}(K)\right) \otimes \mathcal{O}_{q}\left(M_{t, n}(K)\right)$. (Here $K$ is an arbitrary field and $q$ an arbitrary nonzero scalar.) In both cases, the set of coinvariants is a subalgebra of $\mathcal{O}_{q}\left(M_{m, t}(K)\right) \otimes \mathcal{O}_{q}\left(M_{t, n}(K)\right)$, which we identify.
\end{abstract}

\section{Introduction}

One of the highlights of classical invariant theory is the determination of the algebra of invariant functions for the standard action of the general linear group on the variety of pairs of matrices over a field $K$. More precisely, the standard action of $G L_{t}=G L_{t}(K)$ on the variety $V:=M_{m, t}(K) \times M_{t, n}(K)$ induces an action of $G L_{t}$ on $\mathcal{O}(V)$, and the classical theorem determines the algebra of invariants, $\mathcal{O}(V)^{G L_{t}}$. We recall the details below, since, if we assume that $K$ is algebraically closed, the method of proof we follow has an easy geometric translation.

Communicated by M. Kashiwara, October 14, 1999.

1991 Mathematics Subject Classifications: 16W30, 17 B37.

* Department of Mathematics, University of California, Santa Barbara, CA 93106, USA.

** Department of Mathematics, J.C.M.B., Kings Buildings, Mayfield Road, Edinburgh EH9 3JZ, Scotland.

*** Université Jean Monnet (Saint-Étienne), Faculté des Sciences et Techniques, Département de Mathématiques, 23 rue du Docteur Paul Michelon, 42023 Saint-Étienne Cédex 2, France.

The research of the first author was partially supported by National Science Foundation research grant DMS-9622876, and that of the first two authors by NATO Collaborative Research Grant 960250. The first author also thanks Mme. M.-P. Malliavin for inviting him to the Université de Paris VI during January 1999, where a portion of this work was done. Another portion was done while the third author was attending the L.M.S. Symposium on Quantum Groups (Durham, July 1999) and during a subsequent visit to the University of Edinburgh. He wishes to thank the London Mathematical Society for partial financial support of both trips. 
The main theorem of this paper, Theorem 4.5, gives a quantum analog of the above theorem. Since the quantum group $\mathcal{O}_{q}\left(G L_{t}\right)$ is not a group but a Hopf algebra, we first place the classical situation into a Hopf algebra context. This is standard: the action of $G L_{t}$ on $V$ induces a coaction of $\mathcal{O}\left(G L_{t}\right)$ on $\mathcal{O}(V)$, under which $\mathcal{O}(V)$ becomes an $\mathcal{O}\left(G L_{t}\right)$-comodule algebra, and $\mathcal{O}(V)^{G L_{t}}$ equals the algebra of $\mathcal{O}\left(G L_{t}\right)$-coinvariants in $\mathcal{O}(V)$. It is this situation which has a natural quantization: the coordinate ring $\mathcal{O}(V)$ becomes the algebra $\mathcal{O}_{q}(V):=$ $\mathcal{O}_{q}\left(M_{m, t}\right) \otimes \mathcal{O}_{q}\left(M_{t, n}\right)$, and the coaction of $\mathcal{O}\left(G L_{t}\right)$ on $\mathcal{O}(V)$ becomes a coaction of $\mathcal{O}_{q}\left(G L_{t}\right)$ on $\mathcal{O}_{q}(V)$. We prove the First Fundamental Theorem of Coinvariant Theory for this coaction, that is, we identify the set of coinvariants, $\mathcal{O}_{q}(V)^{\operatorname{co} \mathcal{O}_{q}\left(G L_{t}\right)}$

There is a natural comultiplication map $\theta_{q}^{*}: \mathcal{O}_{q}\left(M_{m, n}\right) \rightarrow \mathcal{O}_{q}\left(M_{m, t}\right) \otimes$ $\mathcal{O}_{q}\left(M_{t, n}\right)$, which is the quantum analog of matrix multiplication $M_{m, t} \times M_{t, n} \rightarrow$ $M_{m, n}$. We prove that the set of coinvariants is equal to the image of $\theta_{q}^{*}$. In an earlier paper, [2], the first two authors have shown that the kernel of $\theta_{q}^{*}$ is the ideal generated by the $(t+1) \times(t+1)$ quantum minors of $\mathcal{O}_{q}\left(M_{m, n}\right)$ : this is the Second Fundamental Theorem of Coinvariant Theory for this comodule action. Taken together, these two results give a complete description of $\mathcal{O}_{q}(V)^{\operatorname{co} \mathcal{O}_{q}\left(G L_{t}\right)}$. Further, we investigate the coaction of $\mathcal{O}_{q}\left(S L_{t}\right)$ on $\mathcal{O}_{q}(V)$ induced by that of $\mathcal{O}_{q}\left(G L_{t}\right)$ and identify the coinvariants of this coaction.

The basic structure of our proof follows the outline of one of the possible proofs in the classical invariant theoretical setting. However, there are significant problems that arise due to the noncommutative setting. The most striking one is that, unlike in the commutative case, the $\mathcal{O}_{q}\left(G L_{t}\right)$-comodule $\mathcal{O}_{q}(V)$ is not a comodule algebra. For this reason, it is not even obvious at the outset that the set of coinvariants forms a subalgebra. More generally, the quantum analogs of several maps that we need are not algebra morphisms, and so their properties cannot be analyzed simply by checking how they behave on sets of algebra generators.

Nevertheless, it is useful to start by reviewing the classical situation, to provide a skeleton for our approach.

The classical situation. We fix an algebraically closed field $K$ and positive integers $m, n, t$. For integers $u, v>0$, we write $M_{u, v}=M_{u, v}(K)$ for the set of $u \times v$ matrices with entries in $K$. We will be mainly interested in the general linear group $G L_{t}=G L_{t}(K)$ and its standard action on the algebraic variety $V=$ $V_{m, t, n}:=M_{m, t} \times M_{t, n}$. This action is given by:

$$
\begin{gathered}
G L_{t} \times V \stackrel{\vartheta}{\rightarrow} V \\
(g,(A, B)) \mapsto\left(A g^{-1}, g B\right) .
\end{gathered}
$$

Thus $G L_{t}$ acts on $\mathcal{O}(V) \cong \mathcal{O}\left(M_{m, t}\right) \otimes \mathcal{O}\left(M_{t, n}\right)$. Classical invariant theory is 
interested in computing the subalgebra $\mathcal{O}(V)^{G L_{t}}$ of invariants for this action. The description of this algebra goes as follows. Consider the morphism of varieties

$$
\begin{gathered}
M_{m, t} \times M_{t, n} \stackrel{\theta}{\rightarrow} M_{m, n} \\
(A, B) \mapsto A B
\end{gathered}
$$

and its associated comorphism $\theta^{*}: \mathcal{O}\left(M_{m, n}\right) \rightarrow \mathcal{O}\left(M_{m, t}\right) \otimes \mathcal{O}\left(M_{t, n}\right)$. Let $X_{i j}$ (for $1 \leq i \leq m$ and $1 \leq j \leq n$ ) stand for the usual coordinate functions on the variety $M_{m, n}$, and let $\mathscr{I}_{t+1}$ denote the ideal of $\mathcal{O}\left(M_{m, n}\right)$ generated by all the $(t+1) \times(t+1)$ minors of the generic matrix $\left(X_{i j}\right)$ over $\mathcal{O}\left(M_{m, n}\right)$. (This ideal is zero if $t \geq \min \{m, n\}$.)

Theorem 0.1. The ring of invariants $\mathcal{O}(V)^{G L_{t}}$ equals $\operatorname{Im} \theta^{*}$.

Theorem 0.2. The kernel of $\theta^{*}$ is $\mathscr{I}_{t+1}$.

Theorems 0.1 and 0.2 are respectively known as the First Fundamental Theorem of Invariant Theory and the Second Fundamental Theorem of Invariant Theory (for $G L_{t}$ ). They give a complete description of $\mathcal{O}(V)^{G L_{t}}$.

We denote by $M_{m, n}^{\leq t}$ the subvariety of $M_{m, n}$ of $m \times n$ matrices with rank at most $t$. This variety is just the image of the morphism $\theta$, and so we can factor $\theta$ in the form

$$
M_{m, t} \times M_{t, n} \stackrel{\mu}{\rightarrow} M_{m, n}^{\leq t} \stackrel{\subseteq}{\rightarrow} M_{m, n}
$$

Since the restriction map $r: \mathcal{O}\left(M_{m, n}\right) \rightarrow \mathcal{O}\left(M_{m, n}^{\leq t}\right)$ is surjective, the comorphism $\mu^{*}$ is injective and has the same image as $\theta^{*}$. Thus, Theorem 0.1 can be rephrased in the form $\mathcal{O}(V)^{G L_{t}}=\operatorname{Im} \mu^{*}$. Further, Theorem 0.2 shows that $\operatorname{ker}(r)=\operatorname{ker}\left(\theta^{*}\right)=\mathscr{I}_{t+1}$, and therefore $\mathcal{O}\left(M_{m, n}^{\leq t}\right)=\mathcal{O}\left(M_{m, n}\right) / \mathscr{I}_{t+1}$.

The proof of De Concini and Procesi for Theorem 0.1. We briefly describe the proof of Theorem 0.1 given by De Concini and Procesi in [1]; more precisely, we follow the exposition of that proof given in $[8]$.

The general case can be easily reduced to that where $t<\min \{m, n\}$. So, we restrict attention to that particular case. Let us fix some notation. We will denote by $M_{m, n}^{\leq t, \circ}$ the open subset of $M_{m, n}^{\leq t}$ consisting of matrices with rank at most $t$ whose upper leftmost $t \times t$ minor is nonzero. In a similar way, $M_{m, t}^{\circ}$ denotes the set of $m \times t$ matrices whose uppermost $t \times t$ minor is nonzero, and $M_{t, n}^{\circ}$ denotes the set of $t \times n$ matrices whose leftmost $t \times t$ minor is nonzero. Finally, we set $V^{\circ}=M_{m, t}^{\circ} \times M_{t, n}^{\circ}$. Clearly the action $\gamma$ of $G L_{t}$ on $V$ restricts to an action $\gamma^{\circ}$ on $V^{\circ}$ and we can ask for the invariants of that restricted action. It turns out that they are easy to compute.

Let us adopt the convention that if $(A, B)$ is an element of $V=$ $M_{m, t} \times M_{t, n}$ we write $A$ and $B$ in the forms 


$$
A=\left[\begin{array}{l}
A_{0} \\
A_{1}
\end{array}\right] \quad \text { and } \quad B=\left[\begin{array}{ll}
B_{0} & B_{1}
\end{array}\right]
$$

with $A_{0}, B_{0} \in M_{t}$ while $A_{1} \in M_{m-t, t}$ and $B_{1} \in M_{t, n-t}$.

With these notations, we define a morphism of varieties:

$$
\begin{gathered}
V^{\circ} \stackrel{i}{\rightarrow} M_{m, n}^{\leq t, \circ} \times G L_{t} \\
(A, B) \mapsto\left(A B, B_{0}\right) .
\end{gathered}
$$

It turns out that $i$ is actually an isomorphism of varieties (this is an elementary fact, and the inverse morphism can be explicitly written down) and that the action of $G L_{t}$ on $M_{m, n}^{\leq t, \circ} \times G L_{t}$ induced by $\gamma^{\circ}$ is just the natural one:

$$
\begin{gathered}
G L_{t} \times\left(M_{m, n}^{\leq t, \circ} \times G L_{t}\right) \stackrel{\xi}{\rightarrow} M_{m, n}^{\leq t, \circ} \times G L_{t} \\
(g, C, h) \mapsto(C, g h) .
\end{gathered}
$$

The invariants for $\xi$ are easy to compute, using for instance the coinvariants of the associated $\mathcal{O}\left(G L_{t}\right)$-comodule structure on $\mathcal{O}\left(M_{m, n}^{\leq t, \circ} \times G L_{t}\right)$ (see the quantum case below). One then proves that $\mathcal{O}\left(M_{m, n}^{\leq t, \circ} \times G L_{t}\right) G L_{t}=\mathcal{O}\left(M_{m, n}^{\leq t, \circ}\right) \subseteq$ $\mathcal{O}\left(M_{m, n}^{\leq t, \circ} \times G L_{t}\right)$.

To recover from this the invariants for $\gamma^{\circ}$ it is enough to use the comorphism $i^{*}$ of $i$. Let us consider the morphism

$$
\mu^{\circ}: M_{m, t}^{\circ} \times M_{t, n}^{\circ}=V^{\circ} \rightarrow M_{m, n}^{\leq t, \circ}
$$

given by multiplication of matrices, and denote by $\left(\mu^{\circ}\right)^{*}$ its comorphism:

$$
\left(\mu^{\circ}\right)^{*}: \mathcal{O}\left(M_{m, n}^{\leq t, \circ}\right) \rightarrow \mathcal{O}\left(V^{\circ}\right)=\mathcal{O}\left(M_{m, t}^{\circ}\right) \otimes \mathcal{O}\left(M_{t, n}^{\circ}\right) .
$$

Then we can describe $i^{*}$ as the composition

$$
\begin{aligned}
& i^{*}: \mathcal{O}\left(M_{m, n}^{\leq t, \circ}\right) \otimes \mathcal{O}\left(G L_{t}\right) \stackrel{\left(\mu^{\circ}\right)^{*} \otimes \mathrm{id}}{\longrightarrow} \mathcal{O}\left(V^{\circ}\right) \otimes \mathcal{O}\left(G L_{t}\right) \stackrel{\mathrm{id} \otimes \subseteq}{\longrightarrow} \mathcal{O}\left(V^{\circ}\right) \otimes \mathcal{O}\left(M_{t, n}^{\circ}\right) \\
& \stackrel{=}{\longrightarrow} \mathcal{O}\left(M_{m, t}^{\circ}\right) \otimes \mathcal{O}\left(M_{t, n}^{\circ}\right) \otimes \mathcal{O}\left(M_{t, n}^{\circ}\right) \stackrel{\mathrm{id} \otimes \underline{\mathrm{m}}}{\longrightarrow} \mathcal{O}\left(V^{\circ}\right),
\end{aligned}
$$

where $\underline{\mathrm{m}}$ denotes multiplication in the algebra $\mathcal{O}\left(M_{t, n}^{\circ}\right)$. From the above we get that the ring of invariants for the action $\gamma^{\circ}$ is just $i^{*}\left(\mathcal{O}\left(M_{m, n}^{\leq t, \circ}\right) \otimes 1\right)=$ $\left(\mu^{\circ}\right)^{*}\left(\mathcal{O}\left(M_{m, n}^{\leq t, \circ}\right)\right)$.

The ring of invariants for $\gamma^{\circ}$ can thus be described as the localisation of $\operatorname{Im} \mu^{*}$ with respect to the multiplicative set generated by $d_{Y} \otimes d_{Z}$ where $d_{Y}$ is the uppermost $t \times t$ minor of the generic matrix $\left(Y_{i j}\right)$ of generators of $\mathcal{O}\left(M_{m, t}\right)$, and $d_{Z}$ is the leftmost $t \times t$ minor of the generic matrix $\left(Z_{i j}\right)$ of generators of $\mathcal{O}\left(M_{t, n}\right)$.

The second step of the proof is to show that one can "remove denominators" to deduce invariants for the action $\gamma$ from invariants for the localised 
action $\gamma^{\circ}$. Indeed, if $\phi$ is an invariant function in $\mathcal{O}(V)$ (i.e., for the action $\gamma$ ) then of course it is an invariant function in the localised ring $\mathcal{O}\left(V^{\circ}\right)=$ $\mathcal{O}(V)_{d_{Y} \otimes d_{Z}}$ (for the action $\gamma^{\circ}$ ). Hence, there is a non-negative integer $s$ such that $\phi\left(d_{Y} \otimes d_{Z}\right)^{s} \in \operatorname{Im} \mu^{*}$. Thus, in order to establish that $\mathcal{O}(V)^{G L_{t}}=\operatorname{Im} \mu^{*}$, it is enough to prove that for $\psi \in \mathcal{O}(V)$, if there is a non-negative integer $s$ such that $\psi\left(d_{Y} \otimes d_{Z}\right)^{s} \in \operatorname{Im} \mu^{*}$, then $\psi \in \operatorname{Im} \mu^{*}$. This last result is proved using the theory of standard bases.

In the quantum situation, the lower left quantum minors play a special rôle, since they are normal elements. It is for this reason that, unlike in the commutative case, we will invert leftmost lower $t \times t$ minors instead of leftmost upper ones.

Throughout the paper, we work over an arbitrary base field $K$ and make an arbitrary choice of a nonzero element $q \in K$. We will have to deal with the following four quantized coordinate rings: $\mathcal{O}_{q}\left(M_{m, n}\right), \mathcal{O}_{q}\left(M_{m, t}\right), \mathcal{O}_{q}\left(M_{t, n}\right)$ and $\mathcal{O}_{q}\left(G L_{t}\right)$. In order to avoid confusion, we will denote their respective canonical generators by $X_{i j}, Y_{i j}, Z_{i j}$ and $T_{i j}$. The definitions of these algebras will be as in $[6,2]$; for instance, $X_{i j} X_{i k}=q X_{i k} X_{i j}$ when $j<k$. Thus, each time we use results from [7] we must replace $q$ by $q^{-1}$. Finally, a convention concerning notation: each time that we have to deal with the multiplication map in an algebra, we will denote it by $\underline{\mathrm{m}}$. The context will make clear which algebra is concerned.

\section{$\S 1$. The General Setup}

Below, we will have to deal with the following situation combining a right and a left comodule algebra. Let $(H, \underline{\mathrm{m}}, \eta, \Delta, \varepsilon, S)$ be a Hopf algebra, $\left(A, \rho^{*}\right)$ a right comodule algebra over $H$, and $\left(B, \lambda^{*}\right)$ a left comodule algebra over $H$. Here,

$$
\rho^{*}: A \rightarrow A \otimes H \quad \text { and } \quad \lambda^{*}: B \rightarrow H \otimes B
$$

are the comodule structure maps; that $A$ and $B$ are comodule algebras means that $\rho^{*}$ and $\lambda^{*}$ are also algebra morphisms. It is well known that $A$ can be turned into a left $H$-comodule using the structure map $A \stackrel{\rho^{*}}{\rightarrow} A \otimes H \stackrel{\text { id } \otimes S}{\longrightarrow}$ $A \otimes H \stackrel{\tau_{12}}{\rightarrow} H \otimes A$, where $\tau_{12}$ is the flip. Thus, $A \otimes B$ can be equipped with the structure of a left $H$-comodule via the following structure map:

$$
\begin{gathered}
\gamma^{*}: A \otimes B \stackrel{\rho^{*} \otimes \lambda^{*}}{\longrightarrow} A \otimes H \otimes H \otimes B \stackrel{\text { id } \otimes S \otimes \mathrm{id} \otimes \mathrm{id}}{\longrightarrow} A \otimes H \otimes H \otimes B \\
\stackrel{\tau_{(132)}}{\longrightarrow} H \otimes H \otimes A \otimes B \stackrel{\text { m } \otimes \mathrm{id} \otimes \mathrm{id}}{\longrightarrow} H \otimes A \otimes B,
\end{gathered}
$$


where $\tau_{(132)}$ is the isomorphism which permutes the factors according to the cycle (132), that is, $\tau_{(132)}\left(a \otimes h \otimes h^{\prime} \otimes b\right)=h \otimes h^{\prime} \otimes a \otimes b$. Using the standard comodule notations $\rho^{*}(a)=\sum_{(a)} a_{0} \otimes a_{1}$ and $\lambda^{*}(b)=\sum_{(b)} b_{-1} \otimes b_{0}$ for $a \in A$ and $b \in B$ (cf. [5, p. 11]), one thus has

$$
\gamma^{*}(a \otimes b)=\sum_{(a),(b)} S\left(a_{1}\right) b_{-1} \otimes a_{0} \otimes b_{0} .
$$

Of course, $\left(A \otimes B, \gamma^{*}\right)$ is not a comodule algebra any longer. Nevertheless, this comodule continues to have nice properties that are now described. Let us recall that if $\left(M, v^{*}\right)$ is a left $H$-comodule, then the set of $H$-coinvariants of $\left(M, v^{*}\right)$ (or $v^{*}$-coinvariants for short) is the sub-comodule of $M$ defined by $M^{\text {co } H}:=\left\{x \in M \mid v^{*}(x)=1 \otimes x\right\}$. It is immediate that if $\left(M, v^{*}\right)$ is a comodule algebra then $M^{\text {co } H}$ is a subalgebra of $M$. In the more general situation described above this property is not automatic but still true.

Proposition 1.1. In the above notation:

(a) If $v \in A \otimes B$ is such that $\gamma^{*}(v)=z \otimes v$ for some central element $z \in H$, and if $w \in A \otimes B$ is any element, then $\gamma^{*}(v w)=\gamma^{*}(v) \gamma^{*}(w)$. In particular, this holds when $v$ is a $\gamma^{*}$-coinvariant.

(b) If $v, w \in A \otimes B$ are $\gamma^{*}$-coinvariants, then $v w$ is again a $\gamma^{*}$-coinvariant.

(c) The set $(A \otimes B)^{\mathrm{co} H}$ is a subalgebra of $A \otimes B$.

Proof. Without loss of generality, we may assume that $w=a^{\prime} \otimes b^{\prime}$ is a pure tensor. Moreover, let us write $v=\sum_{i=1}^{r} a_{i} \otimes b_{i}$. Since both $\rho^{*}$ and $\lambda^{*}$ are algebra morphisms, we have

$$
\left(\rho^{*} \otimes \lambda^{*}\right)(v w)=\sum_{i=1}^{r} \sum_{\left(a_{l}\right),\left(b_{l}\right)} \sum_{\left(a^{\prime}\right),\left(b^{\prime}\right)} a_{i, 0} a_{0}^{\prime} \otimes a_{i, 1} a_{1}^{\prime} \otimes b_{i,-1} b_{-1}^{\prime} \otimes b_{i, 0} b_{0}^{\prime},
$$

where $a_{i, 0}=\left(a_{i}\right)_{0}$ etc. Hence,

$$
\begin{aligned}
\left(\gamma^{*}\right)(v w)= & \sum_{i=1}^{r} \sum_{\left(a_{l}\right),\left(b_{l}\right)} \sum_{\left(a^{\prime}\right),\left(b^{\prime}\right)} S\left(a_{i, 1} a_{1}^{\prime}\right) b_{i,-1} b_{-1}^{\prime} \otimes a_{i, 0} a_{0}^{\prime} \otimes b_{i, 0} b_{0}^{\prime} \\
= & \sum_{i=1}^{r} \sum_{\left(a_{l}\right),\left(b_{l}\right)} \sum_{\left(a^{\prime}\right),\left(b^{\prime}\right)} S\left(a_{1}^{\prime}\right) S\left(a_{i, 1}\right) b_{i,-1} b_{-1}^{\prime} \otimes a_{i, 0} a_{0}^{\prime} \otimes b_{i, 0} b_{0}^{\prime} \\
= & \sum_{\left(a^{\prime}\right),\left(b^{\prime}\right)}\left(S\left(a_{1}^{\prime}\right) \otimes 1 \otimes 1\right) \\
& \times\left(\sum_{i=1}^{r} \sum_{\left(a_{1}\right),\left(b_{1}\right)} S\left(a_{i, 1}\right) b_{i,-1} \otimes a_{i, 0} \otimes b_{i, 0}\right)\left(b_{-1}^{\prime} \otimes a_{0}^{\prime} \otimes b_{0}^{\prime}\right) \\
= & \sum_{\left(a^{\prime}\right),\left(b^{\prime}\right)}\left(S\left(a_{1}^{\prime}\right) \otimes 1 \otimes 1\right) \gamma^{*}(v)\left(b_{-1}^{\prime} \otimes a_{0}^{\prime} \otimes b_{0}^{\prime}\right) .
\end{aligned}
$$


But, since $\gamma^{*}(v)=z \otimes v$ and $z$ is central, we have

$$
\begin{aligned}
\left(\gamma^{*}\right)(v w) & =\sum_{\left(a^{\prime}\right),\left(b^{\prime}\right)}\left(S\left(a_{1}^{\prime}\right) \otimes 1 \otimes 1\right)(z \otimes v)\left(b_{-1}^{\prime} \otimes a_{0}^{\prime} \otimes b_{0}^{\prime}\right) \\
& =(z \otimes v) \sum_{\left(a^{\prime}\right),\left(b^{\prime}\right)} S\left(a_{1}^{\prime}\right) b_{-1}^{\prime} \otimes a_{0}^{\prime} \otimes b_{0}^{\prime}=\gamma^{*}(v) \gamma^{*}(w) .
\end{aligned}
$$

This proves statement (a). Clearly, (b) as well as (c) follow at once from (a).

We now record a special case of a coaction for which computing the coinvariants is easy. Again, $(H, \underline{\mathrm{m}}, \eta, \Delta, \varepsilon, S)$ is a Hopf algebra, and $M$ denotes a vector space. Clearly, the map

$$
\begin{gathered}
M \rightarrow H \otimes M \\
a \mapsto 1 \otimes a
\end{gathered}
$$

defines a (trivial) left coaction of $H$ on $M$. Moreover, the comultiplication $\Delta$ of $H$ makes $H$ itself into a left $H$-comodule. The tensor product of these two coactions gives a left coaction of $H$ on $M \otimes H$ :

$$
\begin{gathered}
M \otimes H \stackrel{\xi^{*}}{\rightarrow} H \otimes M \otimes H \\
a \otimes h \mapsto \sum_{(h)} h_{1} \otimes a \otimes h_{2} .
\end{gathered}
$$

Lemma 1.2. With the above notation, $(M \otimes H)^{\mathrm{co} H}=M \otimes 1$.

Proof. Consider $x \in(M \otimes H)^{\operatorname{co} H}$, and write $x=\sum_{i=1}^{s} a_{i} \otimes h_{i}$ where the $a_{i}$ are linearly independent. Now

$$
\sum_{i=1}^{s} \sum_{\left(h_{1}\right)} h_{i, 1} \otimes a_{i} \otimes h_{i, 2}=\xi^{*}(x)=1 \otimes x=\sum_{i=1}^{s} 1 \otimes a_{i} \otimes h_{i} .
$$

Since the $a_{i}$ are linearly independent, we obtain

$$
\Delta\left(h_{i}\right)=\sum_{\left(h_{i}\right)} h_{i, 1} \otimes h_{i, 2}=1 \otimes h_{i}
$$

for each $i$, whence $h_{i}=(\mathrm{id} \otimes \varepsilon) \Delta\left(h_{i}\right)=1 \cdot \varepsilon\left(h_{i}\right)$. This shows that the $h_{i}$ are scalars, and so $x \in M \otimes 1$. Thus, $(M \otimes H)^{\mathrm{co} H} \subseteq M \otimes 1$. The reverse inclusion is obvious.

\section{§2. Quantization of the Standard Action of $G L_{t}$ on $V$}

As in the introduction, we fix our base field $K$ and positive integers $m, n, t$. By analogy with the commutative situation, we put 


$$
\mathcal{O}_{q}(V)=\mathcal{O}_{q}\left(V_{m, t, n}\right):=\mathcal{O}_{q}\left(M_{m, t}\right) \otimes \mathcal{O}_{q}\left(M_{t, n}\right) .
$$

It is easily checked that $\mathcal{O}_{q}(V)$ is an iterated skew polynomial extension of the base field $K$, and so $\mathcal{O}_{q}(V)$ is a noetherian domain. A quantum analogue of the action $\gamma$ is obtained as a coaction of $\mathcal{O}_{q}\left(G L_{t}\right)$ on $\mathcal{O}_{q}(V)$ that we now describe.

It is easy to check that one can define morphisms of algebras satisfying the following rules:

$$
\begin{aligned}
\mathcal{O}_{q}\left(M_{m, t}\right) & \stackrel{\rho_{q}^{*}}{\rightarrow} \mathcal{O}_{q}\left(M_{m, t}\right) \otimes \mathcal{O}_{q}\left(G L_{t}\right) & \mathcal{O}_{q}\left(M_{t, n}\right) \stackrel{\lambda_{q}^{*}}{\rightarrow} \mathcal{O}_{q}\left(G L_{t}\right) \otimes \mathcal{O}_{q}\left(M_{t, n}\right) \\
Y_{i j} & \mapsto \sum_{k=1}^{t} Y_{i k} \otimes T_{k j} & Z_{i j} \mapsto \sum_{k=1}^{t} T_{i k} \otimes Z_{k j} .
\end{aligned}
$$

Moreover, $\rho_{q}^{*}$ endows $\mathcal{O}_{q}\left(M_{m, t}\right)$ with a right $\mathcal{O}_{q}\left(G L_{t}\right)$-comodule algebra structure, while $\lambda_{q}^{*}$ endows $\mathcal{O}_{q}\left(M_{t, n}\right)$ with a left $\mathcal{O}_{q}\left(G L_{t}\right)$-comodule algebra structure. It follows that $\tau_{12} \circ(\mathrm{id} \otimes S) \circ \rho_{q}^{*}$ gives $\mathcal{O}_{q}\left(M_{m, t}\right)$ the structure of a left $\mathcal{O}_{q}\left(G L_{t}\right)$-comodule, where $S$ denotes the antipode in $\mathcal{O}_{q}\left(G L_{t}\right)$. The tensor product of these two left coactions allows us to define a left comodule structure on $\mathcal{O}_{q}(V)$ that we denote by $\gamma_{q}^{*}$. So, as in Section 1 ,

$$
\gamma_{q}^{*}: \mathcal{O}_{q}(V) \rightarrow \mathcal{O}_{q}\left(G L_{t}\right) \otimes \mathcal{O}_{q}(V)
$$

is given by the rule

$$
\gamma_{q}^{*}(a \otimes b)=\sum_{(a),(b)} S\left(a_{1}\right) b_{-1} \otimes a_{0} \otimes b_{0}
$$

for $a \in \mathcal{O}_{q}\left(M_{m, t}\right)$ and $b \in \mathcal{O}_{q}\left(M_{t, n}\right)$.

The main objective of this paper is to calculate $\mathcal{O}_{q}(V)^{\operatorname{co} \mathscr{O}_{q}\left(G L_{t}\right)}$, the set of coinvariants of $\gamma_{q}^{*}$. As we see by Proposition 1.1, this set is a subalgebra of $\mathcal{O}_{q}(V)$.

Quantum minors. Recall that quantum minors in a quantum matrix algebra $\mathcal{O}_{q}\left(M_{u, v}\right)$ correspond to quantum determinants in subalgebras of $\mathcal{O}_{q}\left(M_{u, v}\right)$. More precisely, if $1 \leq r_{1}<r_{2}<\cdots<r_{l} \leq u$ and $1 \leq c_{1}<c_{2}<\cdots<$ $c_{l} \leq v$, then the subalgebra of $\mathcal{O}_{q}\left(M_{u, v}\right)$ generated by $\left\{X_{r_{l} c_{j}} \mid 1 \leq i, j \leq l\right\}$ is naturally isomorphic to $\mathcal{O}_{q}\left(M_{l}\right)$, and the element of this subalgebra corresponding to the quantum determinant in $\mathcal{O}_{q}\left(M_{l}\right)$ is called the quantum minor of $\mathcal{O}_{q}\left(M_{u, v}\right)$ corresponding to the rows $r_{1}, \ldots, r_{l}$ and columns $c_{1}, \ldots, c_{l}$ (see $[6$, $\S 1.2 ; 7, \S 4.3])$. Since we require a number of the formulas and results developed in [2], we shall use the notation of that paper for quantum minors. Thus, the $l \times l$ quantum minor described above will be denoted by the symbol

$$
\left[r_{l} r_{l-1} \cdots r_{1} \mid c_{1} c_{2} \cdots c_{l}\right]
$$

or by $[R \mid C]$ where $R$ and $C$ denote the sets $\left\{r_{1}, \ldots, r_{l}\right\}$ and $\left\{c_{1}, \ldots, c_{l}\right\}$, respectively. 
Remark 2.1. (i) The ideal of $\mathcal{O}_{q}\left(M_{m, n}\right)$ generated by all the $(t+1) \times$ $(t+1)$ quantum minors will be denoted $\mathscr{I}_{t+1}$, or $\mathscr{I}_{t+1}^{m, n}$ when $m$ and $n$ require emphasis. (In case $t \geq \min \{m, n\}$, there are no $(t+1) \times(t+1)$ quantum minors in $\mathcal{O}_{q}\left(M_{m, n}\right)$, and $\mathscr{I}_{t+1}=0$.) Moreover, we put

$$
\mathcal{O}_{q}\left(M_{m, n}^{\leq t}\right):=\mathcal{O}_{q}\left(M_{m, n}\right) / \mathscr{I}_{t+1}^{m, n}
$$

and $x_{i j}:=X_{i j}+\mathscr{I}_{t+1} \in \mathcal{O}_{q}\left(M_{m, n}^{\leq t}\right)$.

(ii) For any $m^{\prime} \geq m$ and $n^{\prime} \geq n$, we will identify $\mathcal{O}_{q}\left(M_{m, n}\right)$ with the subalgebra of $\mathcal{O}_{q}\left(M_{m^{\prime}, n^{\prime}}\right)$ generated by those $X_{i j}$ with $i \leq m$ and $j \leq n$. Now there is a $K$-algebra retraction

$$
\pi_{m, n}=\pi_{m, n}^{m^{\prime}, n^{\prime}}: \mathcal{O}_{q}\left(M_{m^{\prime}, n^{\prime}}\right) \rightarrow \mathcal{O}_{q}\left(M_{m, n}\right)
$$

such that $\pi_{m, n}\left(X_{i j}\right)=X_{i j}$ for $1 \leq i \leq m$ and $1 \leq j \leq n$, and $\pi_{m, n}\left(X_{i j}\right)=0$ for $i>m$ or $j>n$. It is worth mentioning here that the inclusion map $\mathcal{O}_{q}\left(M_{m, n}\right) \rightarrow \mathcal{O}_{q}\left(M_{m^{\prime}, n^{\prime}}\right)$ (corresponding to the identification above) provides a section for $\pi_{m, n}$; this fact will be used without further comment. Note that $\pi_{m, n}[I \mid J]=[I \mid J]$ when $I \subseteq\{1, \ldots, m\}$ and $J \subseteq\{1, \ldots, n\}$, while $\pi_{m, n}[I \mid J]=0$ otherwise. It follows that $\pi_{m, n}\left(\mathscr{I}_{t+1}^{m^{\prime}, n^{\prime}}\right)=\mathscr{I}_{t+1}^{m, n}$, and consequently $\mathscr{I}_{t+1}^{m, n}=$ $\mathscr{I}_{t+1}^{m^{\prime}, n^{\prime}} \cap \mathcal{O}_{q}\left(M_{m, n}\right)$.

(iii) As is easily checked, there is a morphism of algebras

$$
\begin{gathered}
\mathcal{O}_{q}\left(M_{m, n}\right) \stackrel{\theta_{q}^{*}}{\rightarrow} \mathcal{O}_{q}(V) \\
X_{i j} \mapsto \sum_{k=1}^{t} Y_{i k} \otimes Z_{k j} .
\end{gathered}
$$

For instance, this can be checked by using the commutative diagram

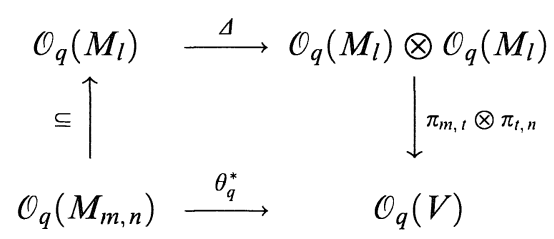

where $l \geq \max \{m, n\}$ and $\Delta$ denotes the comultiplication of $\mathcal{O}_{q}\left(M_{l}\right)$.

(iv) The comultiplication rule for quantum minors in a square quantum matrix algebra, say $\mathcal{O}_{q}\left(M_{l}\right)$, states that

$$
\Delta[I \mid J]=\sum_{\substack{L \subseteq\{1, \ldots, l\} \\|L|=|I|}}[I \mid L] \otimes[L \mid J]
$$

for all quantum minors $[I \mid J] \in \mathcal{O}_{q}\left(M_{l}\right)$ (e.g., $[6,(1.9)]$ ). 
(v) From points (ii), (iii) and (iv), it is clear that $\mathscr{I}_{t+1}$ is contained in the kernel of $\theta_{q}^{*}$. Hence, there is an induced morphism of algebras

$$
\mu_{q}^{*}: \mathcal{O}_{q}\left(M_{m, n}^{\leq t}\right) \rightarrow \mathcal{O}_{q}(V)
$$

such that $\mu_{q}^{*}\left(x_{i j}\right)=\sum_{k=1}^{t} Y_{i k} \otimes Z_{k j}$. The work of the first and second authors in [2] leads to the following result:

Theorem 2.2. In the above notation, the morphism $\mu_{q}^{*}$ is an injection. In particular, $\mathcal{O}_{q}\left(M_{m, n}^{\leq t}\right)$ is a domain.

Proof. We must show that $\operatorname{ker}\left(\theta_{q}^{*}\right)=\mathscr{I}_{t+1}$. In view of Remark 2.1(ii), there is no loss of generality in assuming that $m=n>t$. That case is proved in [2, Proposition 2.4].

Clearly, Theorem 2.2 gives a quantum analogue of the Second Fundamental Theorem of Invariant Theory; see Theorem 0.2 . The rest of this work is devoted to proving a quantum analogue of the First Fundamental Theorem of Invariant Theory; see Theorem 0.1. A first easy proposition is in order.

Proposition 2.3. The set of coinvariants $\mathcal{O}_{q}(V)^{\operatorname{co} \mathcal{O}_{q}\left(G L_{t}\right)}$ is a subalgebra of $\mathcal{O}_{q}(V)$ containing $\operatorname{Im} \mu_{q}^{*}$.

Proof. We are in the setup defined at the begining of Section 1, so we may apply Proposition 1.1(c) to see that $\mathcal{O}_{q}(V)^{\operatorname{co} \mathscr{O}_{q}\left(G L_{t}\right)}$ is a subalgebra of $\mathcal{O}_{q}(V)$. For the rest of the statement, since the map $\mu_{q}^{*}$ is a morphism of algebras, it is enough to prove that $\mu_{q}^{*}\left(x_{i j}\right)$ is a $\gamma_{q}^{*}$-coinvariant, for $1 \leq i \leq m$ and $1 \leq j \leq n$. However,

$$
\begin{aligned}
\gamma_{q}^{*}\left(\mu_{q}^{*}\left(x_{i j}\right)\right) & =\sum_{k=1}^{t} \gamma_{q}^{*}\left(Y_{i k} \otimes Z_{k j}\right)=\sum_{r, s, k=1}^{t} S\left(T_{r k}\right) T_{k s} \otimes Y_{i r} \otimes Z_{s j} \\
& =\sum_{r, s=1}^{t} \varepsilon\left(T_{r s}\right) \otimes Y_{i r} \otimes Z_{s j}=\sum_{r=1}^{t} 1 \otimes Y_{i r} \otimes Z_{r j}=1 \otimes \mu_{q}^{*}\left(x_{i j}\right) .
\end{aligned}
$$

Remark 2.4. By using the retraction maps $\pi_{\boldsymbol{0}, \boldsymbol{\bullet}}$ discussed in Remark 2.1(ii), most results proved for quantized coordinate rings of square matrices immediately carry over to the rectangular case, as in the proof of Theorem 2.2. For the purposes of the present paper, rather than carry over a large number of results in this way, it is more efficient to check that it suffices to prove our main theorem in the case $m=n$. This also allows us to assume that $t<n$, as follows.

Choose an integer $l \geq \max \{m, n\}$ (later, it will be helpful to take $l>$ $\max \{m, n\})$, and observe that we have a commutative diagram as follows: 


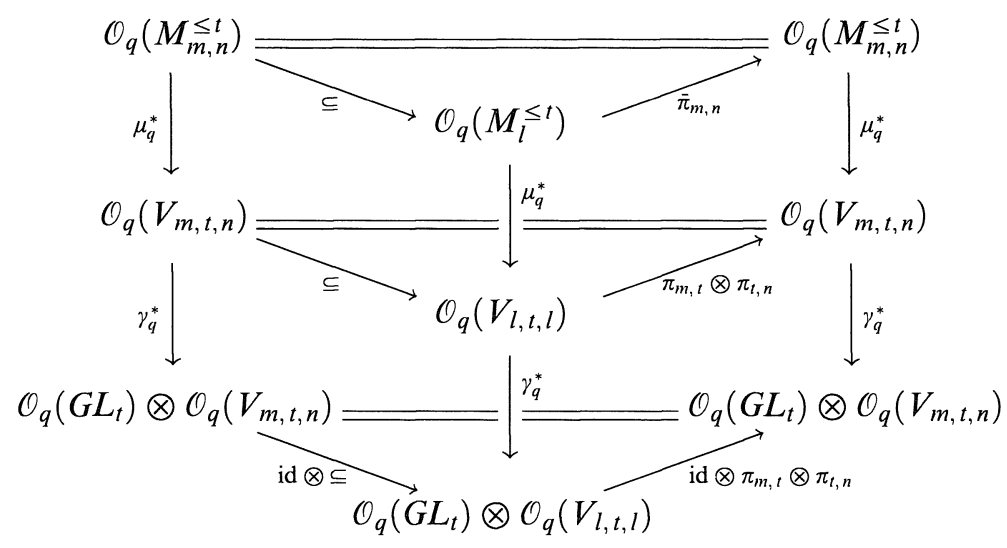

Here ' $\subseteq$ ' is shorthand for various embeddings induced by identifications (cf. Remark 2.1(ii)), and $\bar{\pi}_{m, n}$ denotes the map induced by the retraction $\pi_{m, n}$ : $\mathcal{O}_{q}\left(M_{l}\right) \rightarrow \mathcal{O}_{q}\left(M_{m, n}\right)$ (recall from Remark 2.1(ii) that $\pi_{m, n}\left(\mathscr{I}_{t+1}^{l, l}\right)=\mathscr{I}_{t+1}^{m, n}$ ). It is clear from the above diagram that

$$
\mathcal{O}_{q}\left(V_{l, t, l}\right)^{\operatorname{co} \mathscr{O}_{q}\left(G L_{t}\right)}=\mu_{q}^{*}\left(\mathcal{O}_{q}\left(M_{l}^{\leq t}\right)\right) \Rightarrow \mathcal{O}_{q}\left(V_{m, t, n}\right)^{\operatorname{co} \mathscr{O}_{q}\left(G L_{t}\right)}=\mu_{q}^{*}\left(\mathcal{O}_{q}\left(M_{m, n}^{\leq t}\right)\right) .
$$

Hence, to prove our main theorem it suffices to consider the case $(m=n>t)$.

\section{§3. A Localisation of $\gamma_{q}^{*}$}

In view of Remark 2.4, we assume, until further notice, that $m=n>t$. Certain notations contract slightly in this case; e.g., $\mathcal{O}_{q}\left(M_{m, n}^{\leq t}\right)$ becomes $\mathcal{O}_{q}\left(M_{n}^{\leq t}\right)$.

Remark 3.1. (i) We label the following $t \times t$ quantum minors:

$$
\begin{aligned}
d_{X} & :=[n, \ldots, n-t+1 \mid 1, \ldots, t] \in \mathcal{O}_{q}\left(M_{n}\right) \\
d_{Y} & :=[n, \ldots, n-t+1 \mid 1, \ldots, t] \in \mathcal{O}_{q}\left(M_{n, t}\right) \\
d_{Z} & :=[t, \ldots, 1 \mid 1, \ldots, t] \in \mathcal{O}_{q}\left(M_{t, n}\right) \\
d_{T} & :=[t, \ldots, 1 \mid 1, \ldots, t] \in \mathcal{O}_{q}\left(G L_{t}\right) .
\end{aligned}
$$

Further, we set $d_{x}=d_{X}+\mathscr{I}_{t+1} \in \mathcal{O}_{q}\left(M_{n}^{\leq t}\right)$. It is known that $d_{X} \notin \mathscr{I}_{t+1}$ (for instance, it is clear from Remark $3.2(\mathrm{i})$ below that $\theta_{q}^{*}\left(d_{X}\right) \neq 0$ ), and so $d_{x} \neq 0$.

(ii) It is well known that in any $\mathcal{O}_{q}\left(M_{u, v}\right)$, the "lower left" quantum minors-i.e., those corresponding to the lowest $l$ rows and the leftmost $l$ columns, for any $l$-are normal elements (e.g., see [2, Corollary 5.2]). In particular, the quantum minors $d_{X}, d_{Y}, d_{Z}$ are normal in $\mathcal{O}_{q}\left(M_{n}\right), \mathcal{O}_{q}\left(M_{n, t}\right)$, $\mathcal{O}_{q}\left(M_{t, n}\right)$, respectively. Since $d_{T}$ is the quantum determinant in $\mathcal{O}_{q}\left(G L_{t}\right)$, it is central. It follows from the normality of $d_{X}$ that $d_{x}$ is normal in $\mathcal{O}_{q}\left(M_{n}^{\leq t}\right)$. 
(iii) Since $d_{Y}$ is a normal element in $\mathcal{O}_{q}\left(M_{n, t}\right)$, we can consider the localisation of $\mathcal{O}_{q}\left(M_{n, t}\right)$ with respect to the multiplicative set generated by $d_{Y}$. By analogy with the commutative case, we denote this localisation, $\mathcal{O}_{q}\left(M_{n, t}\right)\left[d_{Y}^{-1}\right]$, by $\mathcal{O}_{q}\left(M_{n, t}^{\circ}\right)$. Similarly, the localisations $\mathcal{O}_{q}\left(M_{t, n}\right)\left[d_{Z}^{-1}\right]$ and $\mathcal{O}_{q}\left(M_{n}^{\leq t}\right)\left[d_{x}^{-1}\right]$ will be denoted $\mathcal{O}_{q}\left(M_{t, n}^{\circ}\right)$ and $\mathcal{O}_{q}\left(M_{n}^{\leq t, \circ}\right)$, respectively. At this stage it is worth mentioning that

$$
\mathcal{O}_{q}\left(M_{n}^{\leq t, \circ}\right) \cong\left(\mathcal{O}_{q}\left(M_{n}\right)\left[d_{X}^{-1}\right]\right) /\left(\mathscr{I}_{t+1}\left[d_{X}^{-1}\right]\right)
$$

this is an easy consequence of the universal properties of quotients and localisations. We will use this isomorphism without further comment.

Remark 3.2. (i) It follows from Remark 2.1(ii,iv) that in the notation of Section 2, one has

$$
\rho_{q}^{*}\left(d_{Y}\right)=d_{Y} \otimes d_{T} \quad \text { and } \quad \lambda_{q}^{*}\left(d_{Z}\right)=d_{T} \otimes d_{Z} .
$$

Further, $\theta_{q}^{*}\left(d_{X}\right)=d_{Y} \otimes d_{Z}$, and so

$$
\mu_{q}^{*}\left(d_{x}\right)=d_{Y} \otimes d_{Z}
$$

(ii) In view of Remark 3.1(iii) and point (i) above, $\rho_{q}^{*}$ and $\lambda_{q}^{*}$ extend uniquely to morphisms of algebras

$$
\begin{aligned}
& \rho_{q}^{* \circ}: \mathcal{O}_{q}\left(M_{n, t}^{\circ}\right) \rightarrow \mathcal{O}_{q}\left(M_{n, t}^{\circ}\right) \otimes \mathcal{O}_{q}\left(G L_{t}\right) \\
& \lambda_{q}^{* \circ}: \mathcal{O}_{q}\left(M_{t, n}^{\circ}\right) \rightarrow \mathcal{O}_{q}\left(G L_{t}\right) \otimes \mathcal{O}_{q}\left(M_{t, n}^{\circ}\right) .
\end{aligned}
$$

(iii) Clearly, $\lambda_{q}^{* \circ}$ defines a left $\mathcal{O}_{q}\left(G L_{t}\right)$-comodule algebra structure on $\mathcal{O}_{q}\left(M_{t, n}^{\circ}\right)$, while $\rho_{q}^{* \circ}$ defines a right $\mathcal{O}_{q}\left(G L_{t}\right)$-comodule algebra structure on $\mathcal{O}_{q}\left(M_{n, t}^{\circ}\right)$. Again, the composition $\tau_{12} \circ(\mathrm{id} \otimes S) \circ \rho_{q}^{* \circ}$ defines a left $\mathcal{O}_{q}\left(G L_{t}\right)$ comodule structure on $\mathcal{O}_{q}\left(M_{n, t}^{\circ}\right)$. Finally, if we set

$$
\mathcal{O}_{q}\left(V^{\circ}\right):=\mathcal{O}_{q}\left(M_{n, t}^{\circ}\right) \otimes \mathcal{O}_{q}\left(M_{t, n}^{\circ}\right)
$$

and tensor the above two left coactions, we obtain a map

$$
\gamma_{q}^{* \circ}: \mathcal{O}_{q}\left(V^{\circ}\right) \rightarrow \mathcal{O}_{q}\left(G L_{t}\right) \otimes \mathcal{O}_{q}\left(V^{\circ}\right),
$$

endowing $\mathcal{O}_{q}\left(V^{\circ}\right)$ with the structure of a left $\mathcal{O}_{q}\left(G L_{t}\right)$-comodule. The map $\gamma_{q}^{* \circ}$ follows the same formula as $\gamma_{q}^{*}$; namely,

$$
\gamma_{q}^{* \circ}(a \otimes b)=\sum_{(a),(b)} S\left(a_{1}\right) b_{-1} \otimes a_{0} \otimes b_{0}
$$

for $a \in \mathcal{O}_{q}\left(M_{n, t}^{\circ}\right)$ and $b \in \mathcal{O}_{q}\left(M_{t, n}^{\circ}\right)$. Obviously, the restriction of $\gamma_{q}^{* \circ}$ to $\mathcal{O}_{q}(V)$ is just $\gamma_{q}^{*}$.

(iv) Recall from Theorem 2.2 that the morphism $\mu_{q}^{*}: \mathcal{O}_{q}\left(M_{n}^{\leq t}\right) \rightarrow \mathcal{O}_{q}(V)$ is injective. Because of Remark 3.1(iii) and point (i) above, we can extend $\mu_{q}^{*}$ to 
the corresponding localised algebras and obtain another injective morphism of algebras:

$$
\mu_{q}^{* \circ}: \mathcal{O}_{q}\left(M_{n}^{\leq t, \circ}\right) \rightarrow \mathcal{O}_{q}\left(V^{\circ}\right) .
$$

We shall need a technical lemma. We have already mentioned that $\gamma_{q}^{*}$ is not an algebra morphism, and so neither is $\gamma_{q}^{* \circ}$. Nevertheless, we have the following statement, the first part of which is a special case of Proposition 1.1.

Lemma 3.3. The set of coinvariants $\mathcal{O}_{q}\left(V^{\circ}\right)^{\operatorname{co} \mathcal{O}_{q}\left(G L_{t}\right)}$ is a subalgebra of $\mathcal{O}_{q}\left(V^{\circ}\right)$, containing $\operatorname{Im} \mu_{q}^{* \circ}$.

Proof. As noted, Proposition 1.1(c) implies that $\mathcal{O}_{q}\left(V^{\circ}\right)^{\operatorname{co} O_{q}\left(G L_{t}\right)}$ is a subalgebra of $\mathcal{O}_{q}\left(V^{\circ}\right)$.

Let us observe that for $r \in \mathbf{N}$, the element $\left(d_{Y} \otimes d_{Z}\right)^{-r}$ is a coinvariant for $\gamma_{q}^{* \circ}$. Indeed, since both $\rho_{q}^{* \circ}$ and $\lambda_{q}^{* \circ}$ are morphisms of algebras, we have $\rho_{q}^{* \circ}\left(d_{Y}^{-r}\right)=d_{Y}^{-r} \otimes d_{T}^{-r}$ and $\lambda_{q}^{* \circ}\left(d_{Z}^{-r}\right)=d_{T}^{-r} \otimes d_{Z}^{-r}$. Recall that $S\left(d_{T}\right)=d_{T}^{-1}[\mathbf{6}$, p. 40; 7, proof of Theorem 5.3.2]. Hence,

$$
\begin{aligned}
\gamma_{q}^{* \circ}\left(\left(d_{Y} \otimes d_{Z}\right)^{-r}\right) & =\gamma_{q}^{* \circ}\left(d_{Y}^{-r} \otimes d_{Z}^{-r}\right)=S\left(d_{T}^{-r}\right) d_{T}^{-r} \otimes d_{Y}^{-r} \otimes d_{Z}^{-r} \\
& =d_{T}^{r} d_{T}^{-r} \otimes d_{Y}^{-r} \otimes d_{Z}^{-r}=1 \otimes d_{Y}^{-r} \otimes d_{Z}^{-r} .
\end{aligned}
$$

This proves the claim. Now since $\mu_{q}^{* \circ}$ is a morphism of algebras, any element $c \in \operatorname{Im} \mu_{q}^{* \circ}$ has the form $c=a\left(d_{Y} \otimes d_{Z}\right)^{-r}$ for some $a \in \operatorname{Im} \mu_{q}^{*}$ and $r \in \mathbf{N}$. So, from the observation above, the fact that $\operatorname{Im} \mu_{q}^{*} \subseteq \mathcal{O}_{q}(V)^{\operatorname{co} \mathcal{O}_{q}\left(G L_{t}\right)}$ (see Proposition 2.3), and the fact that $\mathcal{O}_{q}\left(V^{\circ}\right)^{\operatorname{co} \mathcal{O}_{q}\left(G L_{t}\right)}$ is a subalgebra of $\mathcal{O}_{q}\left(V^{\circ}\right)$, we conclude that $c \in \mathcal{O}_{q}\left(V^{\circ}\right)^{\operatorname{co} O_{q}\left(G L_{t}\right)}$.

Coaction of $\mathcal{O}_{q}\left(G L_{t}\right)$ on $\mathcal{O}_{q}\left(M_{n}^{\leq t, \circ}\right) \otimes \mathcal{O}_{q}\left(G L_{t}\right)$. One key point in the commutative case is that the action of $G L_{t}$ on $V^{\circ}$ turns out to identify with the natural action of $G L_{t}$ on $M_{n}^{\leq t, \circ} \times G L_{t}$ by left translation on the second factor. Here, we define and study a quantum analogue for the latter action.

We can apply the situation described at the end of Section 1 with $M=$ $\mathcal{O}_{q}\left(M_{n}^{\leq t, \circ}\right)$ and $H=\mathcal{O}_{q}\left(G L_{t}\right)$. We thus obtain a left $\mathcal{O}_{q}\left(G L_{t}\right)$-comodule structure on $\mathcal{O}_{q}\left(M_{n}^{\leq t, \circ}\right) \otimes \mathcal{O}_{q}\left(G L_{t}\right)$ via:

$$
\begin{gathered}
\mathcal{O}_{q}\left(M_{n}^{\leq t, \circ}\right) \otimes \mathcal{O}_{q}\left(G L_{t}\right) \stackrel{\xi_{q}^{*}}{\rightarrow} \mathcal{O}_{q}\left(G L_{t}\right) \otimes \mathcal{O}_{q}\left(M_{n}^{\leq t, \circ}\right) \otimes \mathcal{O}_{q}\left(G L_{t}\right) \\
x \otimes h \mapsto \sum_{(h)} h_{1} \otimes x \otimes h_{2} .
\end{gathered}
$$

According to what we proved in Lemma 1.2, we have the following statement:

Proposition 3.4. The set of $\xi_{q}^{*}$-coinvariants is a subalgebra of the algebra $\mathcal{O}_{q}\left(M_{n}^{\leq t, \circ}\right) \otimes \mathcal{O}_{q}\left(G L_{t}\right)$. More precisely,

$$
\left(\mathcal{O}_{q}\left(M_{n}^{\leq t, \circ}\right) \otimes \mathcal{O}_{q}\left(G L_{t}\right)\right)^{\operatorname{co} \mathcal{O}_{q}\left(G L_{t}\right)}=\mathcal{O}_{q}\left(M_{n}^{\leq t, \circ}\right) \otimes 1 .
$$


Link between the coactions $\gamma_{q}^{* \circ}$ and $\xi_{q}^{*}$. In light of the commutative case, one expects that $\mathcal{O}_{q}\left(M_{n}^{\leq t, \circ}\right) \otimes \mathcal{O}_{q}\left(G L_{t}\right)$ and $\mathcal{O}_{q}\left(V^{\circ}\right)$ are isomorphic as $\mathcal{O}_{q}\left(G L_{t}\right)$ comodules. This is, in fact, true, although we do not need the full statement here, only that $\mathcal{O}_{q}\left(V^{\circ}\right)$ is a comodule retraction of $\mathcal{O}_{q}\left(M_{n}^{\leq t, \circ}\right) \otimes \mathcal{O}_{q}\left(G L_{t}\right)$. The morphism that appears as the most natural candidate for an isomorphism between these two comodules is a quantum analog of $i^{*}$ that we describe below.

Remark 3.5. (i) The following injective homomorphisms of algebras will be of constant use in the sequel. One should pay attention to the fact that, due to the choice of the minors we have inverted, these are not the usual maps one might expect to use. These embeddings are all into the lower left corner of the target algebra, in order to make use of the normality of the elements $d_{Y}$ etc. We denote all these morphisms by "inj" in order to avoid introducing too much notation; the context will make clear which one we are using.

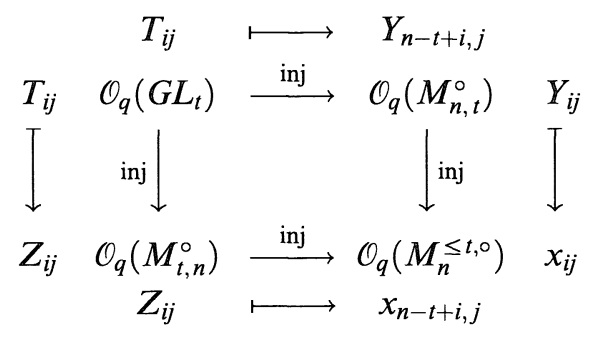

The injectivity of these maps is easy to prove, since in each case we can exhibit a left inverse. We show how to deal with the case of the left hand one; similar arguments hold for the others. The retraction $\pi_{t, t}$ (see Remark 2.1(ii)) induces a morphism of algebras $\mathcal{O}_{q}\left(M_{t, n}^{\circ}\right) \rightarrow \mathcal{O}_{q}\left(G L_{t}\right)$ that maps $Z_{i j}$ to $T_{i j}$ if $j \leq t$ and to 0 if $j>t$. Clearly, this map provides a left inverse for inj.

(ii) Identify $\mathcal{O}_{q}\left(M_{n, t}^{\circ}\right)$ (respectively, $\mathcal{O}_{q}\left(M_{t, n}^{\circ}\right)$ ) with its image in $\mathcal{O}_{q}\left(M_{n}^{\leq t, \circ}\right)$. Then, the restriction of $\mu_{q}^{* \circ}$ to $\mathcal{O}_{q}\left(M_{n, t}^{\circ}\right)$ (respectively, $\mathcal{O}_{q}\left(M_{t, n}^{\circ}\right)$ ) coincides with $\rho_{q}^{* \circ}$ (respectively, $\lambda_{q}^{* \circ}$ ), as one can easily check on generators (this is enough since the maps involved are morphisms of algebras). More precisely, we are claiming that the following diagram is commutative:

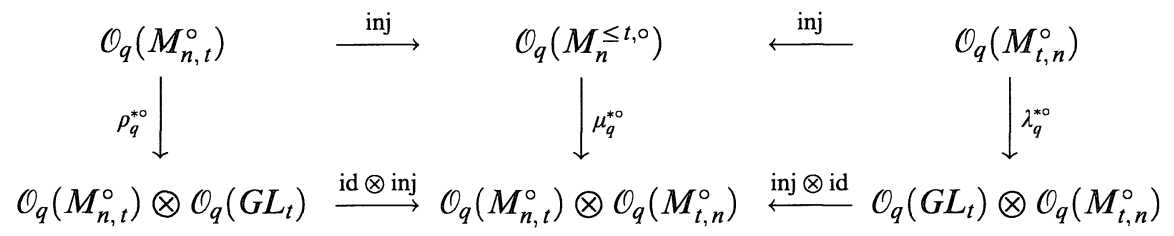

For example, to see that the left hand square commutes, it suffices to check that $\mu_{q}^{* \circ} \circ$ inj and $\left(\mathrm{id} \otimes\right.$ inj) $\circ \rho_{q}^{* \circ}$ agree on the $Y_{i j}$ and on $d_{Y}$. This is clear-both maps send $Y_{i j}$ to $\sum_{k=1}^{t} Y_{i k} \otimes Z_{k j}$ and $d_{Y}$ to $d_{Y} \otimes d_{Z}$. The commutativity of the right hand square is proved analogously. 
(iii) Finally, the restriction of $\lambda_{q}^{* \circ}$ (respectively, $\left.\mu_{q}^{* \circ}\right)$ to $\mathcal{O}_{q}\left(G L_{t}\right)$ coincides with $\Delta$, i.e., the following diagram is commutative:

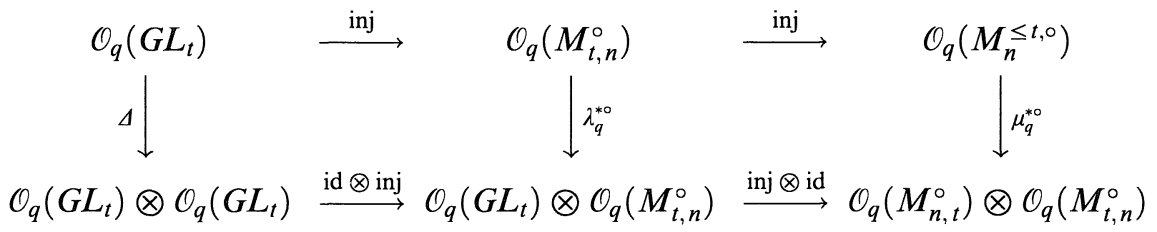

In the following, we will identify the algebras $\mathcal{O}_{q}\left(G L_{t}\right), \mathcal{O}_{q}\left(M_{n, t}^{\circ}\right), \mathcal{O}_{q}\left(M_{t, n}^{\circ}\right)$ with their images under inj and we will use the commutativity of the above diagrams without explicit mention.

Quantization of $i^{*}$. Due to our identification of $\mathcal{O}_{q}\left(G L_{t}\right)$ with a subalgebra of $\mathcal{O}_{q}\left(M_{t, n}^{\circ}\right)$, we can use the multiplication map $\underline{\mathrm{m}}$ on $\mathcal{O}_{q}\left(M_{t, n}^{\circ}\right)$ to endow $\mathcal{O}_{q}\left(M_{t, n}^{\circ}\right)$ with the structure of a right $\mathcal{O}_{q}\left(G L_{t}\right)$-module. We now consider the following quantum analogue of $i^{*}$ :

$$
\begin{aligned}
i_{q}^{*}: \mathcal{O}_{q}\left(M_{n}^{\leq t, \circ}\right) \otimes \mathcal{O}_{q}\left(G L_{t}\right) & \stackrel{\mu_{q}^{* \circ} \otimes \mathrm{id}}{\longrightarrow} \mathcal{O}_{q}\left(M_{n, t}^{\circ}\right) \otimes \mathcal{O}_{q}\left(M_{t, n}^{\circ}\right) \otimes \mathcal{O}_{q}\left(G L_{t}\right) \\
& \stackrel{\operatorname{id} \otimes \underline{\mathrm{m}}}{\longrightarrow} \mathcal{O}_{q}\left(M_{n, t}^{\circ}\right) \otimes \mathcal{O}_{q}\left(M_{t, n}^{\circ}\right)=\mathcal{O}_{q}\left(V^{\circ}\right) .
\end{aligned}
$$

It can be proved that $i_{q}^{*}$ is an isomorphism of left $\mathcal{O}_{q}\left(G L_{t}\right)$-comodules, but we shall not need the full result-for our purposes, it suffices to construct a right inverse for $i_{q}^{*}$.

Recall that the antipode $S$ in $\mathcal{O}_{q}\left(G L_{t}\right)$ is bijective (e.g., [7, Theorem 5.4.2]).

Consider the map $j_{q}^{*}: \mathcal{O}_{q}\left(V^{\circ}\right) \rightarrow \mathcal{O}_{q}\left(M_{n}^{\leq t, \circ}\right) \otimes \mathcal{O}_{q}\left(G L_{t}\right)$ defined as the composition of the following maps:

$$
\begin{aligned}
\mathcal{O}_{q}\left(V^{\circ}\right) & =\mathcal{O}_{q}\left(M_{n, t}^{\circ}\right) \otimes \mathcal{O}_{q}\left(M_{t, n}^{\circ}\right) \stackrel{\rho_{q}^{* \circ} \otimes \lambda_{q}^{* \circ}}{\longrightarrow} \mathcal{O}_{q}\left(M_{n, t}^{\circ}\right) \otimes \mathcal{O}_{q}\left(G L_{t}\right) \otimes \mathcal{O}_{q}\left(G L_{t}\right) \otimes \mathcal{O}_{q}\left(M_{t, n}^{\circ}\right) \\
& \stackrel{\mathrm{id} \otimes \mathrm{id} \otimes \Delta \otimes \mathrm{id}}{\longrightarrow} \mathcal{O}_{q}\left(M_{n, t}^{\circ}\right) \otimes \mathcal{O}_{q}\left(G L_{t}\right) \otimes \mathcal{O}_{q}\left(G L_{t}\right) \otimes \mathcal{O}_{q}\left(G L_{t}\right) \otimes \mathcal{O}_{q}\left(M_{t, n}^{\circ}\right) \\
& \stackrel{\mathrm{id} \otimes S \otimes \mathrm{id} \otimes S^{-1} \otimes \mathrm{id}}{\longrightarrow} \mathcal{O}_{q}\left(M_{n, t}^{\circ}\right) \otimes \mathcal{O}_{q}\left(G L_{t}\right) \otimes \mathcal{O}_{q}\left(G L_{t}\right) \otimes \mathcal{O}_{q}\left(G L_{t}\right) \otimes \mathcal{O}_{q}\left(M_{t, n}^{\circ}\right) \\
& \stackrel{\tau_{(135)(24)}}{\longrightarrow} \mathcal{O}_{q}\left(M_{t, n}^{\circ}\right) \otimes \mathcal{O}_{q}\left(G L_{t}\right) \otimes \mathcal{O}_{q}\left(M_{n, t}^{\circ}\right) \otimes \mathcal{O}_{q}\left(G L_{t}\right) \otimes \mathcal{O}_{q}\left(G L_{t}\right) \\
& \stackrel{\mathrm{inj} \otimes \mathrm{inj} \otimes \mathrm{inj} \otimes \underline{\mathrm{m}}}{\longrightarrow} \mathcal{O}_{q}\left(M_{n}^{\leq t, \circ}\right) \otimes \mathcal{O}_{q}\left(M_{n}^{\leq t, \circ}\right) \otimes \mathcal{O}_{q}\left(M_{n}^{\leq t, \circ}\right) \otimes \mathcal{O}_{q}\left(G L_{t}\right) \\
& \stackrel{\underline{\mathrm{m}}(\underline{\mathrm{m}} \otimes \mathrm{id}) \otimes \mathrm{id}}{\longrightarrow} \mathcal{O}_{q}\left(M_{n}^{\leq t, \circ}\right) \otimes \mathcal{O}_{q}\left(G L_{t}\right) .
\end{aligned}
$$

Here $\tau_{(135)(24)}$ denotes the isomorphism which shuffles the factors via the permutation $(135)(24)$, that is,

$$
\tau_{(135)(24)}\left(x_{1} \otimes x_{2} \otimes x_{3} \otimes x_{4} \otimes x_{5}\right)=x_{5} \otimes x_{4} \otimes x_{1} \otimes x_{2} \otimes x_{3} .
$$


For a pure tensor $a \otimes b \in \mathcal{O}_{q}\left(V^{\circ}\right)$, we can give the following formula for $j_{q}^{*}(a \otimes b)$ using the notation $\rho_{q}^{*}(a)=\sum_{(a)} a_{0} \otimes a_{1}$ and $(\Delta \otimes \mathrm{id}) \lambda_{q}^{*}(b)=$ $\sum_{(b)} b_{-2} \otimes b_{-1} \otimes b_{0} . \quad$ Namely,

$$
j_{q}^{*}(a \otimes b)=\sum_{(a),(b)} b_{0} S^{-1}\left(b_{-1}\right) a_{0} \otimes S\left(a_{1}\right) b_{-2} .
$$

Lemma 3.6. The map $j_{q}^{*}$ is a morphism of left $\mathcal{O}_{q}\left(G L_{t}\right)$-comodules.

Proof. We must prove that the following diagram is commutative:

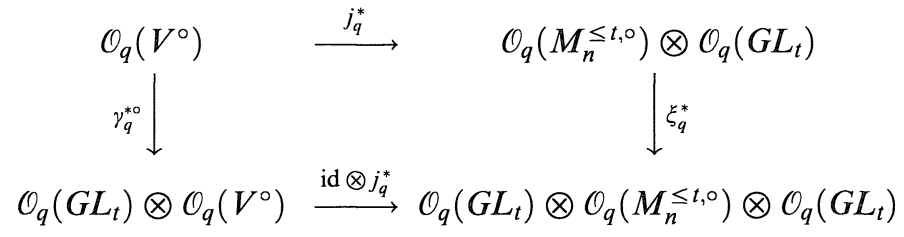

If $a \in \mathcal{O}_{q}\left(M_{n, t}^{\circ}\right)$ and $b \in \mathcal{O}_{q}\left(M_{t, n}^{\circ}\right)$, then (with the usual conventions for expanding components)

$$
\begin{aligned}
\left(\mathrm{id} \otimes j_{q}^{*}\right) \gamma_{q}^{* \circ}(a \otimes b) & =\sum_{(a),(b)} S\left(a_{1}\right) b_{-1} \otimes j_{q}^{*}\left(a_{0} \otimes b_{0}\right) \\
& =\sum_{(a),(b)} S\left(a_{2}\right) b_{-3} \otimes b_{0} S^{-1}\left(b_{-1}\right) a_{0} \otimes S\left(a_{1}\right) b_{-2}
\end{aligned}
$$

Since $\Delta(S(c) d)=\sum_{(c),(d)} S\left(c_{2}\right) d_{1} \otimes S\left(c_{1}\right) d_{2}$ for $c, d \in \mathcal{O}_{q}\left(G L_{t}\right)$, we also have

$$
\begin{aligned}
\xi_{q}^{*} j_{q}^{*}(a \otimes b) & =\sum_{(a),(b)} \xi_{q}^{*}\left(b_{0} S^{-1}\left(b_{-1}\right) a_{0} \otimes S\left(a_{1}\right) b_{-2}\right) \\
& =\sum_{(a),(b)} S\left(a_{2}\right) b_{-3} \otimes b_{0} S^{-1}\left(b_{-1}\right) a_{0} \otimes S\left(a_{1}\right) b_{-2} .
\end{aligned}
$$

Therefore $\left(\mathrm{id} \otimes j_{q}^{*}\right) \gamma_{q}^{* \circ}(a \otimes b)=\xi_{q}^{*} j_{q}^{*}(a \otimes b)$, as desired.

In the sequel, we shall need the identity

$$
\sum_{(c)} c_{2} S^{-1}\left(c_{1}\right)=\sum_{(c)} S^{-1}\left(c_{2}\right) c_{1}=\varepsilon(c) \cdot 1
$$

for $c \in \mathcal{O}_{q}\left(G L_{t}\right)$. This follows from the defining property of the antipode $S$ by applying the antiautomorphism $S^{-1}$. Moreover, in view of the identity $\Delta \circ S=\tau_{12} \circ(S \otimes S) \circ \Delta$ (e.g., [5, Proposition 1.5.10]), we also have

$$
\left(S^{-1} \otimes S^{-1}\right) \circ \tau_{12} \circ \Delta=\Delta \circ S^{-1} \text {. }
$$

Lemma 3.7. The composition $i_{q}^{*} \circ j_{q}^{*}$ is the identity map on $\mathcal{O}_{q}\left(V^{\circ}\right)$. 
Proof. Let $a \otimes b \in \mathcal{O}_{q}\left(V^{\circ}\right)$. Using Remark 3.5(ii,iii) and the fact that $\mu_{q}^{* \circ}$ is a morphism of algebras, we have

$$
\begin{aligned}
\left(\mu_{q}^{* \circ} \otimes \mathrm{id}\right) j_{q}^{*}(a \otimes b) & =\sum_{(a),(b)}\left(\mu_{q}^{* \circ}\left(b_{0}\right)\left(\mu_{q}^{* \circ} S^{-1}\left(b_{-1}\right)\right) \mu_{q}^{* \circ}\left(a_{0}\right)\right) \otimes S\left(a_{1}\right) b_{-2} \\
& =\sum_{(a),(b)}\left(\lambda_{q}^{* \circ}\left(b_{0}\right)\left(\Delta S^{-1}\left(b_{-1}\right)\right) \rho_{q}^{* \circ}\left(a_{0}\right)\right) \otimes S\left(a_{1}\right) b_{-2} \\
& =\sum_{(a),(b)}\left(\left(b_{-1} \otimes b_{0}\right)\left(\Delta S^{-1}\left(b_{-2}\right)\right)\left(a_{0} \otimes a_{1}\right)\right) \otimes S\left(a_{2}\right) b_{-3} .
\end{aligned}
$$

Consequently, with the help of the identities above, we see that

$$
\begin{aligned}
\left(\mu_{q}^{* \circ} \otimes \mathrm{id}\right) j_{q}^{*}(a \otimes b) & =\sum_{(a),(b)} b_{-1} S^{-1}\left(b_{-2}\right) a_{0} \otimes b_{0} S^{-1}\left(b_{-3}\right) a_{1} \otimes S\left(a_{2}\right) b_{-4} \\
& =\sum_{(a),(b)} \varepsilon\left(b_{-1}\right) a_{0} \otimes b_{0} S^{-1}\left(b_{-2}\right) a_{1} \otimes S\left(a_{2}\right) b_{-3} \\
& =\sum_{(a),(b)} a_{0} \otimes b_{0} S^{-1}\left(b_{-2} \varepsilon\left(b_{-1}\right)\right) a_{1} \otimes S\left(a_{2}\right) b_{-3} \\
& =\sum_{(a),(b)} a_{0} \otimes b_{0} S^{-1}\left(b_{-1}\right) a_{1} \otimes S\left(a_{2}\right) b_{-2} .
\end{aligned}
$$

Thus,

$$
\begin{aligned}
i_{q}^{*} j_{q}^{*}(a \otimes b) & =\sum_{(a),(b)} a_{0} \otimes b_{0} S^{-1}\left(b_{-1}\right) a_{1} S\left(a_{2}\right) b_{-2}=\sum_{(a),(b)} a_{0} \otimes b_{0} S^{-1}\left(b_{-1}\right) \varepsilon\left(a_{1}\right) b_{-2} \\
& =\sum_{(a),(b)} a_{0} \varepsilon\left(a_{1}\right) \otimes b_{0} S^{-1}\left(b_{-1}\right) b_{-2}=\sum_{(b)} a \otimes b_{0} \varepsilon\left(b_{-1}\right)=a \otimes b .
\end{aligned}
$$

Proposition 3.8. $\mathcal{O}_{q}\left(V^{\circ}\right)^{\operatorname{co} \mathscr{C}_{q}\left(G L_{t}\right)}=\operatorname{Im} \mu_{q}^{* \circ}$.

Proof. We have the inclusion " $\supseteq$ " by Lemma 3.3. To prove the reverse inclusion, consider an arbitrary $\gamma_{q}^{* \circ}$-coinvariant $c \in \mathcal{O}_{q}\left(V^{\circ}\right)$. Since $j_{q}^{*}$ is a comodule morphism (Lemma 3.6), $j_{q}^{*}(c)$ is a $\xi_{q}^{*}$-coinvariant in $\mathcal{O}_{q}\left(M_{n}^{\leq t, \circ}\right) \otimes$ $\mathcal{O}_{q}\left(G L_{t}\right)$. Hence, $j_{q}^{*}(c) \in \mathcal{O}_{q}\left(M_{n}^{\leq t, \circ}\right) \otimes 1$ by Proposition 3.4. Since $i_{q}^{*} j_{q}^{*}(c)=c$ by Lemma 3.7 , and since clearly $i_{q}^{*}\left(\mathcal{O}_{q}\left(M_{n}^{\leq t, \circ}\right) \otimes 1\right)=\operatorname{Im} \mu_{q}^{* \circ}$, we conclude that $c \in \operatorname{Im} \mu_{q}^{* \circ}$. This verifies the inclusion " $\subseteq$ ".

\section{§4. The First Fundamental Theorem for $\mathcal{O}_{q}\left(G L_{t}\right)$-coinvariants}

In this section, we complete the proof of our main theorem-that the set of $\gamma_{q}^{*}$-coinvariants in $\mathcal{O}_{q}(V)$ equals the image of $\mu_{q}^{*}$. We first discuss how to obtain $\gamma_{q}^{*}$-coinvariants from $\gamma_{q}^{* \circ}$-coinvariants by "removing denominators". 
Remark 4.1. (i) As we already mentioned in Remark 3.2(iii), the restriction of $\gamma_{q}^{* \circ}$ to $\mathcal{O}_{q}(V)$ is just $\gamma_{q}^{*}$. So, if $w \in \mathcal{O}_{q}(V)$ is a $\gamma_{q}^{*}$-coinvariant, it is also a $\gamma_{q}^{* \circ}$-coinvariant. Thus, according to Proposition 3.8, $w \in \operatorname{Im} \mu_{q}^{* \circ}$. This means that there exists $s \in \mathbf{N}$ such that $w\left(d_{Y} \otimes d_{Z}\right)^{s} \in \operatorname{Im} \mu_{q}^{*}$. So, to prove that the set of $\gamma_{q}^{*}$-coinvariants equals $\operatorname{Im} \mu_{q}^{*}$, it is enough to show the following property (cf. Proposition 2.3):

$$
\forall w \in \mathcal{O}_{q}(V), \quad \text { if } w\left(d_{Y} \otimes d_{Z}\right) \in \operatorname{Im} \mu_{q}^{*}, \quad \text { then } w \in \operatorname{Im} \mu_{q}^{*} .
$$

(ii) Recall from Remark 3.2(i) that the image under $\mu_{q}^{*}$ of the (normal) element $d_{x} \in \mathcal{O}_{q}\left(M_{n}^{\leq t}\right)$ is the (normal) element $d_{Y} \otimes d_{Z} \in \mathcal{O}_{q}(V)$. We claim that to prove $(\dagger)$, it is enough (in fact, equivalent) to verify the following property:

$$
\left(\mu_{q}^{*}\right)^{-1}\left(\left\langle d_{Y} \otimes d_{Z}\right\rangle\right)=\left\langle d_{x}\right\rangle .
$$

Thus, assume that $(\ddagger)$ holds, and let $w \in \mathcal{O}_{q}(V)$ such that $w\left(d_{Y} \otimes d_{Z}\right) \in \operatorname{Im} \mu_{q}^{*}$. Then $w\left(d_{Y} \otimes d_{Z}\right)=\mu_{q}^{*}(y)$ for some $y \in \mathcal{O}_{q}\left(M_{n}^{\leq t}\right)$, and $(\ddagger)$ implies that $y=z d_{x}$ for some $z \in \mathcal{O}_{q}\left(M_{n}^{\leq t}\right)$. Since $\mu_{q}^{*}$ is a morphism of algebras, $w\left(d_{Y} \otimes d_{Z}\right)=$ $\mu_{q}^{*}(y)=\mu_{q}^{*}(z)\left(d_{Y} \otimes d_{Z}\right)$, and so $w=\mu_{q}^{*}(z)$ because $\mathcal{O}_{q}(V)$ is a domain. This shows that $(\ddagger)$ implies $(\dagger)$. The converse follows easily from the injectivity of $\mu_{q}^{*}$ (see Theorem 2.2).

Remark 4.2. (i) The computations in this section and in Section 6 below rely on the preferred bases for the algebras $\mathcal{O}_{q}\left(M_{n}\right), \mathcal{O}_{q}\left(M_{n, t}\right)$, and $\mathcal{O}_{q}\left(M_{t, n}\right)$ developed in [2, Section 1], and we follow the notation of that paper. See, in particular, [2, Corollary 1.11] for the rectangular case. We recall the notation $\left[T \mid T^{\prime}\right]$ for the product of quantum minors corresponding to an allowable bitableau $\left(T, T^{\prime}\right)$, and we recall also that it is sometimes convenient to label rows of $\left(T, T^{\prime}\right)$ in the form $(I, J)$ where $I$ and $J$ are sets of row and column indices, respectively.

(ii) On tensoring the preferred basis elements of $\mathcal{O}_{q}\left(M_{n, t}\right)$ with those of $\mathcal{O}_{q}\left(M_{t, n}\right)$, we obtain a preferred basis for $\mathcal{O}_{q}(V)$ consisting of all pure tensors $\left[S \mid S^{\prime}\right] \otimes\left[T \mid T^{\prime}\right]$ where $\left(S, S^{\prime}\right)$ and $\left(T, T^{\prime}\right)$ are preferred bitableaux with appropriate entries. In particular, $S$ and $T$ each have at most $t$ columns.

(iii) Because of the quantum Laplace expansions [6, Proposition 1.1 and Corollary; 7, Corollary 4.4.4], the ideal $\mathscr{I}_{t+1}$ in $\mathcal{O}_{q}\left(M_{n}\right)$ contains all the $r \times r$ quantum minors for $t+1 \leq r \leq n$. Hence, $\left[T \mid T^{\prime}\right] \in \mathscr{I}_{t+1}$ whenever $\left(T, T^{\prime}\right)$ is a preferred bitableau and $T$ has at least $t+1$ columns.

The statement and proof of [2, Proposition 2.4] show that $\mathscr{I}_{t+1}$ does not contain any nonzero linear combinations of distinct products $\left[S \mid S^{\prime}\right]$ where the $\left(S, S^{\prime}\right)$ are preferred bitableaux and $S$ has at most $t$ columns. Consequently, $\mathscr{I}_{t+1}$ has a $K$-basis consisting of the products $\left[T \mid T^{\prime}\right]$ such that $\left(T, T^{\prime}\right)$ is a preferred bitableau and $T$ has at least $t+1$ columns.

(iv) In view of point (iii), the cosets $\left[T \mid T^{\prime}\right]+\mathscr{I}_{t+1}$ such that $\left(T, T^{\prime}\right)$ is a 
preferred bitableau and $T$ has at most $t$ columns form a $K$-basis for $\mathcal{O}_{q}\left(M_{n}^{\leq t}\right)$. To simplify the notation, we shall write cosets $a+\mathscr{I}_{t+1}$ in the form $\bar{a}$. Thus, $\overline{\left[T \mid T^{\prime}\right]}=0$ whenever $T$ has more than $t$ columns.

It is convenient to label two special index sets:

$$
\tilde{I}=\{n-t+1, \ldots, n\} \quad \text { and } \quad \tilde{J}=\{1, \ldots, t\} .
$$

With this notation, observe that $d_{X}$ and $d_{Y}$ can both be labeled $[\tilde{I} \mid \tilde{J}]$, while $d_{Z}$ and $d_{T}$ can both be labeled $[\tilde{J} \mid \tilde{J}]$.

Lemma 4.3. (a) A K-basis for $\left\langle d_{Y}\right\rangle$ consists of all $\left[T \mid T^{\prime}\right\}$ in $\mathcal{O}_{q}\left(M_{n, t}\right)$ where $\left(T, T^{\prime}\right)$ is a preferred bitableau with first row $(\tilde{I}, \tilde{J})$.

(b) A K-basis for $\left\langle d_{Z}\right\rangle$ consists of all $\left[T \mid T^{\prime}\right]$ in $\mathcal{O}_{q}\left(M_{t, n}\right)$ where $\left(T, T^{\prime}\right)$ is a preferred bitableau with first row $(\tilde{J}, \tilde{J})$.

(c) A K-basis for $\left\langle d_{Y} \otimes d_{Z}\right\rangle$ consists of all pure tensors $\left[S \mid S^{\prime}\right] \otimes\left[T \mid T^{\prime}\right]$ in $\mathcal{O}_{q}(V)$ where $\left(S, S^{\prime}\right)$ and $\left(T, T^{\prime}\right)$ are preferred bitableaux with first rows $(\tilde{I}, \tilde{J})$ and $(\tilde{J}, \tilde{J})$, respectively.

(d) A K-basis for $\left\langle d_{x}\right\rangle$ consists of all $\overline{\left[T \mid T^{\prime}\right]}$ in $\mathcal{O}_{q}\left(M_{n}^{\leq t}\right)$ where $\left(T, T^{\prime}\right)$ is a preferred bitableau with first row $(\tilde{I}, \tilde{J})$.

Proof. (a) Obviously any such $\left[T \mid T^{\prime}\right]$ lies in $\left\langle d_{Y}\right\rangle$, because $d_{Y}=[\tilde{I} \mid \tilde{J}]$. Recall that $d_{Y}$ is a normal element of $\mathcal{O}_{q}\left(M_{n, t}\right)$, so that $\left\langle d_{Y}\right\rangle=d_{Y} \mathcal{O}_{q}\left(M_{n, t}\right)$. Since $(\tilde{I}, \tilde{J})$ is the minimum index pair labeling quantum minors in $\mathcal{O}_{q}\left(M_{n, t}\right)$, the preferred basis of $\mathcal{O}_{q}\left(M_{n, t}\right)$ is closed under left multiplication by $d_{Y}$. Hence, $\left\langle d_{Y}\right\rangle$ is spanned by products $d_{Y}\left[S \mid S^{\prime}\right]$ as $\left(S, S^{\prime}\right)$ runs through all preferred bitableaux, and each such $d_{Y}\left[S \mid S^{\prime}\right]=\left[T \mid T^{\prime}\right]$ for some preferred bitableau $\left(T, T^{\prime}\right)$ with first row $(\tilde{I}, \tilde{J})$.

(b) This is proved in the same manner as part (a).

(c) Note that because $d_{Y}$ and $d_{Z}$ are normal elements of $\mathcal{O}_{q}\left(M_{n, t}\right)$ and $\mathcal{O}_{q}\left(M_{t, n}\right)$, respectively,

$$
\left\langle d_{Y} \otimes d_{Z}\right\rangle=\left(d_{Y} \otimes d_{Z}\right) \mathcal{O}_{q}(V)=d_{Y} \mathcal{O}_{q}\left(M_{n, t}\right) \otimes d_{Z} \mathcal{O}_{q}\left(M_{t, n}\right)=\left\langle d_{Y}\right\rangle \otimes\left\langle d_{Z}\right\rangle .
$$

Hence, part (c) follows directly from parts (a) and (b).

(d) By Remark 4.2(iv), a $K$-basis for $\mathcal{O}_{q}\left(M_{n}^{\leq t}\right)$ consists of all $\overline{\left[T \mid T^{\prime}\right]}$ such that $\left(T, T^{\prime}\right)$ is a preferred bitableau and $T$ has at most $t$ columns. Among index pairs $(I, J)$ with $|I| \leq t$, the minimum element is $(\tilde{I}, \tilde{J})$. Since $d_{x}=\overline{[\tilde{I} \mid \tilde{J}]}$, we therefore obtain part (d) in exactly the same manner as part (a).

In the following proof, we shall need the natural multigradings on quantum matrix algebras (cf. $[2, \S 1.5]$ ). For instance, $\mathcal{O}_{q}\left(M_{n, t}\right)$ is graded by $\mathbb{Z}^{n} \times \mathbb{Z}^{t}$ with each generator $Y_{i j}$ having degree $\left(\varepsilon_{i}, \varepsilon_{j}\right)$, where $\varepsilon_{1}, \varepsilon_{2}, \ldots$ denote the standard basis elements in $\mathbf{Z}^{n}$ and $\mathbf{Z}^{t}$. In the proof, we use the label 'homogeneous' to refer to homogeneous elements with respect to the above gradings. 
Proposition 4.4. $\left(\mu_{q}^{*}\right)^{-1}\left(\left\langle d_{Y} \otimes d_{Z}\right\rangle\right)=\left\langle d_{x}\right\rangle$.

Proof. The inclusion ' $\supseteq$ ' is clear since $\mu_{q}^{*}\left(d_{x}\right)=d_{Y} \otimes d_{Z}$. If this inclusion is proper, choose an element

$$
x=\sum_{i=1}^{r} \alpha_{i} \overline{\left[T_{i} \mid T_{i}^{\prime}\right]} \in\left(\mu_{q}^{*}\right)^{-1}\left(\left\langle d_{Y} \otimes d_{Z}\right\rangle\right) \backslash\left\langle d_{x}\right\rangle
$$

where the $\alpha_{i}$ are nonzero scalars, the $\left(T_{i}, T_{i}^{\prime}\right)$ are distinct preferred bitableaux, and the $T_{i}$ have at most $t$ columns. We may assume that none of the $\overline{\left[T_{i} \mid T_{i}^{\prime}\right]}$ lie in $\left\langle d_{x}\right\rangle$. Thus, by Lemma $4.3(\mathrm{~d})$, none of the $\left(T_{i}, T_{i}^{\prime}\right)$ has first row $(\tilde{I}, \tilde{J})$. Define $n$-tuples $\bar{\rho}\left(T_{i}\right)$ as in $[2, \S 2.2]$, and let $\bar{\rho}$ be the minimum of the $\bar{\rho}\left(T_{i}\right)$ under reverse lexicographic order.

After re-indexing, we may assume that there is some $r^{\prime}$ such that $\bar{\rho}\left(T_{i}\right)=\bar{\rho}$ for $i \leq r^{\prime}$ and $\bar{\rho}\left(T_{i}\right)>_{\text {rlex }} \bar{\rho}$ for $i>r^{\prime}$. Applying [2, Lemma 2.3] to each $\mu_{q}^{*} \overline{\left[T_{i} \mid T_{i}^{\prime}\right]}\left(=\theta_{q}^{*}\left[T_{i} \mid T_{i}^{\prime}\right]\right)$ and collecting terms, we see (using the notation of $[2$, $\S 2.2])$ that

$$
\mu_{q}^{*}(x)=\sum_{i=1}^{r^{\prime}} \alpha_{i}\left[T_{i} \mid \mu\left(T_{i}\right)\right] \otimes\left[\mu^{\prime}\left(T_{i}\right) \mid T_{i}^{\prime}\right]+\sum_{j} X_{j} \otimes Y_{j}
$$

where the $X_{j}$ and $Y_{j}$ are homogeneous with $\bar{c}\left(X_{j}\right)=\bar{r}\left(Y_{j}\right)>_{\text {rlex }} \bar{\rho}$. We then observe (as in the proof of [2, Proposition 2.4]) that all of the $X_{j}$ belong to different homogeneous components than the $\left[T_{i} \mid \mu\left(T_{i}\right)\right]$ for $i \leq r^{\prime}$. Since $\mu_{q}^{*}(x) \in\left\langle d_{Y} \otimes d_{Z}\right\rangle=\left\langle d_{Y}\right\rangle \otimes\left\langle d_{Z}\right\rangle$ and the ideal $\left\langle d_{Y}\right\rangle$ is homogeneous, it follows that

$$
\sum_{i=1}^{r^{\prime}} \alpha_{i}\left[T_{i} \mid \mu\left(T_{i}\right)\right] \otimes\left[\mu^{\prime}\left(T_{i}\right) \mid T_{i}^{\prime}\right] \in\left\langle d_{Y} \otimes d_{Z}\right\rangle .
$$

For $1 \leq i<j \leq r^{\prime}$, either $T_{i} \neq T_{j}$ or $T_{i}^{\prime} \neq T_{j}^{\prime}$, whence $\left(T_{i}, \mu\left(T_{i}\right)\right) \neq$ $\left(T_{j}, \mu\left(T_{j}\right)\right)$ or $\left(\mu^{\prime}\left(T_{i}\right), T_{i}^{\prime}\right) \neq\left(\mu^{\prime}\left(T_{j}\right), T_{j}^{\prime}\right)$. Recall from $[2, \S 2.2]$ that the $\left(T_{i}, \mu\left(T_{i}\right)\right)$ and the $\left(\mu^{\prime}\left(T_{i}\right), T_{i}^{\prime}\right)$ are preferred bitableaux. In view of Lemma 4.3(c), it follows that $T_{i}$ has first row $\tilde{I}$ and $T_{i}^{\prime}$ has first row $\tilde{J}$ for $1 \leq i \leq r^{\prime}$. However, this contradicts our choices above, and therefore the proposition is proved.

Theorem 4.5. Let $m, n, t$ be arbitrary positive integers. The set of $\gamma_{q}^{*}$-coinvariants in $\mathcal{O}_{q}(V)=\mathcal{O}_{q}\left(M_{m, t}\right) \otimes \mathcal{O}_{q}\left(M_{t, n}\right)$ is $\operatorname{Im} \mu_{q}^{*}$, that is,

$$
\left(\mathcal{O}_{q}\left(M_{m, t}\right) \otimes \mathcal{O}_{q}\left(M_{t, n}\right)\right)^{\operatorname{co} \mathcal{O}_{q}\left(G L_{t}\right)}=\mu_{q}^{*}\left(\mathcal{O}_{q}\left(M_{m, n}^{\leq t}\right)\right) .
$$

Proof. In view of Remark 2.4, there is no loss of generality in assuming that $m=n>t$. The theorem is then immediate using Remark 4.1 and Proposition 4.4. 


\section{§5. $\mathcal{O}_{q}\left(S L_{t}\right)$-coinvariants}

For arbitrary positive integers $m, n, t$, there is a natural coaction $\Gamma_{q}^{*}$ of $\mathcal{O}_{q}\left(S L_{t}\right)$ on $\mathcal{O}_{q}(V)$ induced from the $\mathcal{O}_{q}\left(G L_{t}\right)$-coaction $\gamma_{q}^{*}$ that we have been studying. Denote by $\pi$ the natural quotient map $\mathcal{O}_{q}\left(G L_{t}\right) \rightarrow \mathcal{O}_{q}\left(S L_{t}\right)$. The coaction $\Gamma_{q}^{*}: \mathcal{O}_{q}(V) \rightarrow \mathcal{O}_{q}\left(S L_{t}\right) \otimes \mathcal{O}_{q}(V)$ is given by $\Gamma_{q}^{*}:=(\pi \otimes \mathrm{id}) \circ \gamma_{q}^{*}$. In this section and the next, we compute the $\Gamma_{q}^{*}$-coinvariants.

In fact, $\Gamma_{q}^{*}$ can be constructed from the left coaction of $\mathcal{O}_{q}\left(S L_{t}\right)$ on $\mathcal{O}_{q}\left(M_{t, n}\right)$ given by

$$
(\pi \otimes \mathrm{id}) \circ \lambda_{q}^{*}: \mathcal{O}_{q}\left(M_{t, n}\right) \rightarrow \mathcal{O}_{q}\left(S L_{t}\right) \otimes \mathcal{O}_{q}\left(M_{t, n}\right)
$$

and the right coaction of $\mathcal{O}_{q}\left(S L_{t}\right)$ on $\mathcal{O}_{q}\left(M_{m, t}\right)$ given by

$$
(\mathrm{id} \otimes \pi) \circ \rho_{q}^{*}: \mathcal{O}_{q}\left(M_{m, t}\right) \rightarrow \mathcal{O}_{q}\left(M_{m, t}\right) \otimes \mathcal{O}_{q}\left(S L_{t}\right)
$$

by using the construction described in Section 1 .

Proposition 5.1. The set of coinvariants $\mathcal{O}_{q}(V)^{\operatorname{co} \mathcal{O}_{q}\left(S L_{t}\right)}$ is a subalgebra of $\mathcal{O}_{q}(V)$ containing $\operatorname{Im} \mu_{q}^{*}$.

Proof. That $\mathcal{O}_{q}(V)^{\operatorname{co} \mathcal{O}_{q}\left(S L_{t}\right)}$ is a subalgebra of $\mathcal{O}_{q}(V)$ follows from Proposition 1.1(c). It contains $\operatorname{Im} \mu_{q}^{*}$ because the elements of $\operatorname{Im} \mu_{q}^{*}$ are $\gamma_{q}^{*}$ coinvariants and hence also $\Gamma_{q}^{*}$-coinvariants.

In order to describe the $\Gamma_{q}^{*}$-coinvariants, we may assume that $m=n$ and that $t<n$, as in Remark 2.4. We start by observing that there is a natural $\mathbf{Z}$-grading on quantum matrices which will simplify the problem. The algebra $\mathcal{O}_{q}\left(M_{n, t}\right)$ can be graded by total degree in the variables $Y_{i j}$ and we put $\mathcal{O}_{q}\left(M_{n, t}\right)=\bigoplus_{i \geq 0} \mathcal{O}_{q}\left(M_{n, t}\right)_{i}$, where $\mathcal{O}_{q}\left(M_{n, t}\right)_{i}$ is the subspace spanned by monomials of total degree $i$ in $\mathcal{O}_{q}\left(M_{n, t}\right)$. In the same way, and with obvious notation, $\mathcal{O}_{q}\left(M_{t, n}\right)=\bigoplus_{j \geq 0} \mathcal{O}_{q}\left(M_{t, n}\right)_{j}$. It follows that

$$
\mathcal{O}_{q}(V)=\bigoplus_{i, j=0}^{\infty} \mathcal{O}_{q}(V)_{i, j},
$$

where $\mathcal{O}_{q}(V)_{i, j}=\mathcal{O}_{q}\left(M_{n, t}\right)_{i} \otimes \mathcal{O}_{q}\left(M_{t, n}\right)_{j}$.

Recall that $\rho_{q}^{*}: \mathcal{O}_{q}\left(M_{n, t}\right) \rightarrow \mathcal{O}_{q}\left(M_{n, t}\right) \otimes \mathcal{O}_{q}\left(G L_{t}\right)$ is the algebra morphism defined by $\rho_{q}^{*}\left(Y_{i j}\right)=\sum_{k=1}^{t} Y_{i k} \otimes T_{k j}$. Thus, it is clear that $\rho_{q}^{*}\left(\mathcal{O}_{q}\left(M_{n, t}\right)_{i}\right) \subseteq$ $\mathcal{O}_{q}\left(M_{n, t}\right)_{i} \otimes \mathcal{O}_{q}\left(G L_{t}\right)$. Similarly, one has $\lambda_{q}^{*}\left(\mathcal{O}_{q}\left(M_{t, n}\right)_{j}\right) \subseteq \mathcal{O}_{q}\left(G L_{t}\right) \otimes \mathcal{O}_{q}\left(M_{t, n}\right)_{j}$. It follows that

$$
\begin{aligned}
& \gamma_{q}^{*}\left(\mathcal{O}_{q}(V)_{i, j}\right) \subseteq \mathcal{O}_{q}\left(G L_{t}\right) \otimes \mathcal{O}_{q}(V)_{i, j} \\
& \Gamma_{q}^{*}\left(\mathcal{O}_{q}(V)_{i, j}\right) \subseteq \mathcal{O}_{q}\left(S L_{t}\right) \otimes \mathcal{O}_{q}(V)_{i, j}
\end{aligned}
$$

for all $i, j$. 
Given $w \in \mathcal{O}_{q}(V)$, we will write $w=\sum_{i, j} w_{i j}$ with $w_{i j} \in \mathcal{O}_{q}(V)_{i, j}$.

Lemma 5.2. Let $w=\sum_{i, j} w_{i j} \in \mathcal{O}_{q}(V)$. Then

(a) $w$ is a $\gamma_{q}^{*}$-coinvariant if and only if each $w_{i j}$ is a $\gamma_{q}^{*}$-coinvariant.

(b) $w$ is a $\Gamma_{q}^{*}$-coinvariant if and only if each $w_{i j}$ is a $\Gamma_{q}^{*}$-coinvariant.

Proof. If each $w_{i j}$ is a $\gamma_{q}^{*}$-coinvariant then, obviously, $w$ is a $\gamma_{q}^{*}$-coinvariant. Conversely, suppose that $w$ is a $\gamma_{q}^{*}$-coinvariant. One has $\sum_{i, j} 1 \otimes w_{i j}$ $=1 \otimes w=\gamma_{q}^{*}(w)=\sum_{i, j} \gamma_{q}^{*}\left(w_{i j}\right)$, and from the discussion above, we see that $\gamma_{q}^{*}\left(w_{i j}\right) \in \mathcal{O}_{q}\left(G L_{t}\right) \otimes \mathcal{O}_{q}(V)_{i, j}$. Thus, since $\mathcal{O}_{q}\left(G L_{t}\right) \otimes \mathcal{O}_{q}(V)=\bigoplus_{i, j} \mathcal{O}_{q}\left(G L_{t}\right) \otimes$ $\mathcal{O}_{q}(V)_{i, j}$, one has $\gamma_{q}^{*}\left(w_{i j}\right)=1 \otimes w_{i j}$ for all $i, j$. This finishes the proof of $(\mathrm{a})$; the proof of (b) is similar.

The previous result shows that, in order to describe the $\Gamma_{q}^{*}$-coinvariants, it is enough to describe the coinvariants which are in each $\mathcal{O}_{q}(V)_{i, j}$. It is obvious from the definition of $\Gamma_{q}^{*}$ that any $\gamma_{q}^{*}$-coinvariant will be a $\Gamma_{q}^{*}$ coinvariant. Our first aim is to show that any homogeneous $\Gamma_{q}^{*}$-coinvariant $w$ is a $\gamma_{q}^{*}$-semi-coinvariant, meaning that $\gamma_{q}^{*}(w)=d_{T}^{s} \otimes w$ for some integer $s$; this is achieved in Theorem 5.5. We begin by constructing some maps which will help us to prove this fact.

The above grading on $\mathcal{O}_{q}(V)$ relates to the following comodule structure. Consider the Hopf algebra $\mathcal{O}_{q}\left(K^{\times}\right)=K\left[T^{ \pm 1}\right]$ and the algebra morphism $\pi^{\prime}$ : $\mathcal{O}_{q}\left(G L_{t}\right) \rightarrow \mathcal{O}_{q}\left(K^{\times}\right)$such that $T_{i i} \mapsto T$ for all $i$, while $T_{i j} \mapsto 0$ for $i \neq j$. It is easily checked that $\pi^{\prime}$ is, in fact, a morphism of Hopf algebras. The composite map

$$
\mathcal{O}_{q}(V) \stackrel{\gamma_{q}^{*}}{\longrightarrow} \mathcal{O}_{q}\left(G L_{t}\right) \otimes \mathcal{O}_{q}(V) \stackrel{\pi^{\prime} \otimes \mathrm{id}}{\longrightarrow} \mathcal{O}_{q}\left(K^{\times}\right) \otimes \mathcal{O}_{q}(V)
$$

thus gives $\mathcal{O}_{q}(V)$ the structure of a left comodule over $\mathcal{O}_{q}\left(K^{\times}\right)$. The following proposition shows that, in fact, $\mathcal{O}_{q}(V)$ is an $\mathcal{O}_{q}\left(K^{\times}\right)$-comodule algebra.

Proposition 5.3. (a) $\left(\pi^{\prime} \otimes \mathrm{id}\right) \circ \gamma_{q}^{*}$ is an algebra morphism.

(b) $\left(\pi^{\prime} \otimes \mathrm{id}\right) \gamma_{q}^{*}\left(Y_{i j} \otimes 1\right)=T^{-1} \otimes Y_{i j} \otimes 1$ and $\left(\pi^{\prime} \otimes \mathrm{id}\right) \gamma_{q}^{*}\left(1 \otimes Z_{i j}\right)=T \otimes$ $1 \otimes Z_{i j}$ for all $i, j$.

(c) $\left(\pi^{\prime} \otimes \mathrm{id}\right) \gamma_{q}^{*}(w)=T^{j-i} \otimes w$ for all $w \in \mathcal{O}_{q}(V)_{i, j}$.

Proof. (a) Recall that, for $a \in \mathcal{O}_{q}\left(M_{n, t}\right)$ and $b \in \mathcal{O}_{q}\left(M_{t, n}\right)$, if we put $\quad \rho_{q}^{*}(a)=\sum_{(a)} a_{0} \otimes a_{1} \quad$ and $\quad \lambda_{q}^{*}(b)=\sum_{(b)} b_{-1} \otimes b_{0}, \quad$ then $\quad \gamma_{q}^{*}(a \otimes b)=$ $\sum_{(a),(b)} S\left(a_{1}\right) b_{-1} \otimes a_{0} \otimes b_{0}$. If, also, $a^{\prime} \otimes b^{\prime} \in \mathcal{O}_{q}(V)$ then, since $\rho_{q}^{*}$ and $\lambda_{q}^{*}$ are algebra morphisms, one has

$$
\gamma_{q}^{*}\left((a \otimes b)\left(a^{\prime} \otimes b^{\prime}\right)\right)=\sum_{(a),(b),\left(a^{\prime}\right),\left(b^{\prime}\right)} S\left(a_{1}^{\prime}\right) S\left(a_{1}\right) b_{-1} b_{-1}^{\prime} \otimes a_{0} a_{0}^{\prime} \otimes b_{0} b_{0}^{\prime}
$$


Using the fact that $\mathcal{O}_{q}\left(K^{\times}\right)$is a commutative algebra we then get $\left(\pi^{\prime} \otimes \mathrm{id}\right) \gamma_{q}^{*}\left((a \otimes b)\left(a^{\prime} \otimes b^{\prime}\right)\right)=\left(\left(\pi^{\prime} \otimes \mathrm{id}\right) \gamma_{q}^{*}\right)(a \otimes b)\left(\left(\pi^{\prime} \otimes \mathrm{id}\right) \gamma_{q}^{*}\right)\left(a^{\prime} \otimes b^{\prime}\right)$.

(b) If $A_{j k}$ denotes the $(t-1) \times(t-1)$ quantum minor of $\mathcal{O}_{q}\left(G L_{t}\right)$ obtained by deleting row $j$ and column $k$, then $S\left(T_{k j}\right)=(-q)^{k-j} A_{j k} d_{T}^{-1}$, by [7, Theorem 5.3.2], and so

$$
\gamma_{q}^{*}\left(Y_{i j} \otimes 1\right)=\sum_{k=1}^{t} S\left(T_{k j}\right) \otimes Y_{i k} \otimes 1=\sum_{k=1}^{t}(-q)^{k-j} A_{j k} d_{T}^{-1} \otimes Y_{i k} \otimes 1
$$

and $\gamma_{q}^{*}\left(1 \otimes Z_{i j}\right)=\sum_{k=1}^{t} T_{i k} \otimes 1 \otimes Z_{k j} . \quad$ It follows that $\left(\pi^{\prime} \otimes \mathrm{id}\right) \gamma_{q}^{*}\left(Y_{i j} \otimes 1\right)=$ $T^{-1} \otimes Y_{i j} \otimes 1$ and that $\left(\pi^{\prime} \otimes \mathrm{id}\right) \gamma_{q}^{*}\left(1 \otimes Z_{i j}\right)=T \otimes 1 \otimes Z_{i j}$.

(c) This is clear from parts (a) and (b).

We shall need the algebra morphism

$$
\phi_{q}^{*}: \mathcal{O}_{q}\left(G L_{t}\right) \otimes \mathcal{O}_{q}\left(K^{\times}\right) \rightarrow \mathcal{O}_{q}\left(G L_{t}\right)[\bar{\chi}]:=\mathcal{O}_{q}\left(G L_{t}\right)[\chi] /\left\langle\chi^{t}-d_{T}^{-1}\right\rangle,
$$

where $\chi$ is an indeterminate and $\bar{\chi}=\chi+\left\langle\chi^{t}-d_{T}^{-1}\right\rangle$, such that each $T_{i j} \otimes 1 \mapsto$ $T_{i j} \bar{\chi}$ and $1 \otimes T \mapsto \bar{\chi}^{-1}$. It is easy to check that $\phi_{q}^{*}\left(d_{T} \otimes 1\right)=d_{T} \bar{\chi}^{t}=1$, and so there is an induced algebra morphism

$$
\varphi_{q}^{*}: \mathcal{O}_{q}\left(S L_{t}\right) \otimes \mathcal{O}_{q}\left(K^{\times}\right) \rightarrow \mathcal{O}_{q}\left(G L_{t}\right)[\bar{\chi}]
$$

such that $\varphi_{q}^{*} \circ(\pi \otimes \mathrm{id})=\phi_{q}^{*}$.

Finally, let $\alpha_{q}: \mathcal{O}_{q}\left(G L_{t}\right) \rightarrow \mathcal{O}_{q}\left(G L_{t}\right)[\bar{\chi}]$ denote the canonical injection.

Lemma 5.4. (a) $\phi_{q}^{*} \circ\left(\mathrm{id} \otimes \pi^{\prime}\right) \circ \Delta=\alpha_{q}$.

(b) $\quad\left(\varphi_{q}^{*} \otimes \mathrm{id}\right) \circ\left(\mathrm{id} \otimes \pi^{\prime} \otimes \mathrm{id}\right) \circ\left(\mathrm{id} \otimes \gamma_{q}^{*}\right) \circ \Gamma_{q}^{*}=\left(\alpha_{q} \otimes \mathrm{id}\right) \circ \gamma_{q}^{*}$.

Proof. (a) It is enough to check this on the generators $T_{i j}$, since each of the maps involved is an algebra morphism. One has

$$
\phi_{q}^{*}\left(\mathrm{id} \otimes \pi^{\prime}\right) \Delta\left(T_{i j}\right)=\phi_{q}^{*}\left(\mathrm{id} \otimes \pi^{\prime}\right)\left(\sum_{k=1}^{t} T_{i k} \otimes T_{k j}\right)=\phi_{q}^{*}\left(T_{i j} \otimes T\right)=T_{i j}
$$

(b) Since $\gamma_{q}^{*}$ is the structure map of a left $\mathcal{O}_{q}\left(G L_{t}\right)$-comodule, we have $(\Delta \otimes \mathrm{id}) \circ \gamma_{q}^{*}=\left(\mathrm{id} \otimes \gamma_{q}^{*}\right) \circ \gamma_{q}^{*}$. In view of part $(\mathrm{a})$, it follows that

$$
\begin{aligned}
\left(\alpha_{q} \otimes \mathrm{id}\right) \circ \gamma_{q}^{*} & =\left(\phi_{q}^{*} \otimes \mathrm{id}\right) \circ\left(\mathrm{id} \otimes \pi^{\prime} \otimes \mathrm{id}\right) \circ(\Delta \otimes \mathrm{id}) \circ \gamma_{q}^{*} \\
& =\left(\phi_{q}^{*} \otimes \mathrm{id}\right) \circ\left(\mathrm{id} \otimes \pi^{\prime} \otimes \mathrm{id}\right) \circ\left(\mathrm{id} \otimes \gamma_{q}^{*}\right) \circ \gamma_{q}^{*}
\end{aligned}
$$

We also have the following commutative diagram: 


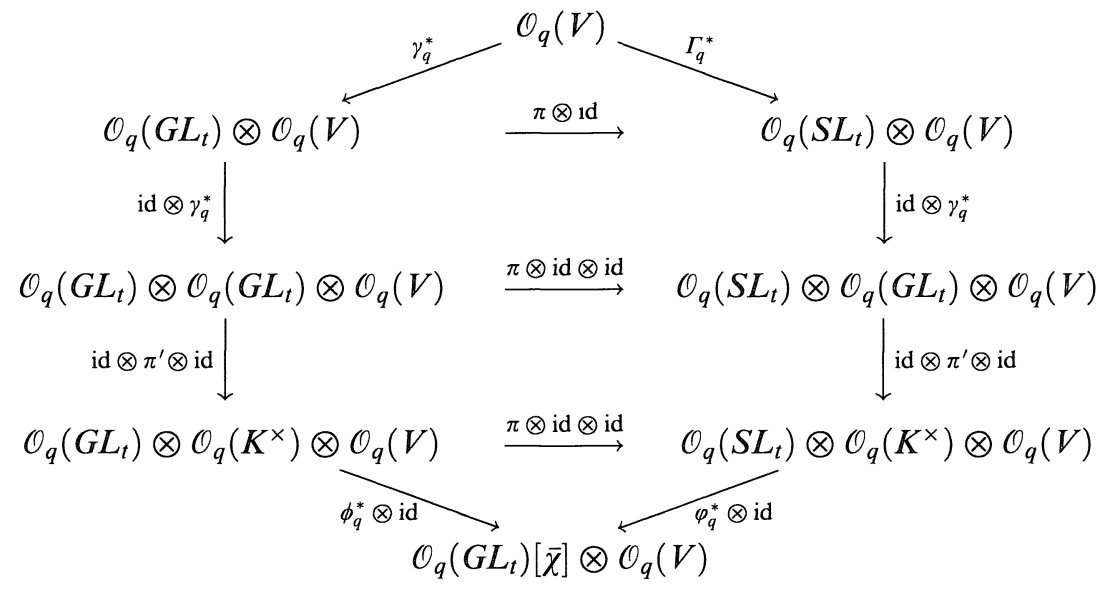

Thus $\quad\left(\phi_{q}^{*} \otimes \mathrm{id}\right) \circ\left(\mathrm{id} \otimes \pi^{\prime} \otimes \mathrm{id}\right) \circ\left(\mathrm{id} \otimes \gamma_{q}^{*}\right) \circ \gamma_{q}^{*}=\left(\varphi_{q}^{*} \otimes \mathrm{id}\right) \circ\left(\mathrm{id} \otimes \pi^{\prime} \otimes \mathrm{id}\right) \circ$ $\left(\mathrm{id} \otimes \gamma_{q}^{*}\right) \circ \Gamma_{q}^{*}$, which completes the proof of part (b).

Theorem 5.5. Let $w \in \mathcal{O}_{q}(V)_{i, j}$ be a $\Gamma_{q}^{*}$-coinvariant.

(a) The difference $i-j$ is divisible by $t$, and $\gamma_{q}^{*}(w)=d_{T}^{-s} \otimes w$ where $s=$ $(i-j) / t$.

(b) If $i \geq j$, then $\left(1 \otimes d_{Z}^{s}\right) w$ is a $\gamma_{q}^{*}$-coinvariant.

(c) If $i \leq j$, then $\left(d_{Y}^{-s} \otimes 1\right) w$ is a $\gamma_{q}^{*}$-coinvariant.

Proof. (a) By Proposition 5.3(c), we have $\left(\pi^{\prime} \otimes \mathrm{id}\right) \gamma_{q}^{*}(w)=T^{j-i} \otimes w$. From this and the assumption that $\Gamma_{q}^{*}(w)=1 \otimes w$, it follows that $\left(\varphi_{q}^{*} \otimes \mathrm{id}\right)\left(\mathrm{id} \otimes \pi^{\prime} \otimes \mathrm{id}\right)\left(\mathrm{id} \otimes \gamma_{q}^{*}\right) \Gamma_{q}^{*}(w)=\left(\varphi_{q}^{*} \otimes \mathrm{id}\right)\left(\mathrm{id} \otimes \pi^{\prime} \otimes \mathrm{id}\right)\left(\mathrm{id} \otimes \gamma_{q}^{*}\right)(1 \otimes w)$

$$
=\left(\varphi_{q}^{*} \otimes \mathrm{id}\right)\left(1 \otimes T^{j-i} \otimes w\right)=\bar{\chi}^{i-j} \otimes w .
$$

Thus, by Lemma 5.4(b), $\left(\alpha_{q} \otimes \mathrm{id}\right) \gamma_{q}^{*}(w)=\bar{\chi}^{i-j} \otimes w$. In particular,

$$
\bar{\chi}^{i-j} \otimes w \in \operatorname{Im}\left(\alpha_{q} \otimes \mathrm{id}\right)=\mathcal{O}_{q}\left(G L_{t}\right) \cdot 1 \otimes \mathcal{O}_{q}(V) .
$$

Since $\mathcal{O}_{q}\left(G L_{t}\right)[\bar{\chi}]$ is a free $\mathcal{O}_{q}\left(G L_{t}\right)$-module with basis $\left\{1, \bar{\chi}, \ldots, \bar{\chi}^{t-1}\right\}$, we have $t \mid i-j$. Set $s=(i-j) / t$. Now

$$
\left(\alpha_{q} \otimes \mathrm{id}\right) \gamma_{q}^{*}(w)=\bar{\chi}^{t s} \otimes w=\left(\alpha_{q} \otimes \mathrm{id}\right)\left(d_{T}^{-s} \otimes w\right),
$$

and therefore $\gamma_{q}^{*}(w)=d_{T}^{-s} \otimes w$, as required.

(b) Since $\lambda_{q}^{*}$ is an algebra morphism, we see using Remark 3.2(i) that $\lambda_{q}^{*}\left(d_{Z}^{s}\right)=d_{T}^{s} \otimes d_{Z}^{s}$, and hence $\gamma_{q}^{*}\left(1 \otimes d_{Z}^{s}\right)=d_{T}^{s} \otimes 1 \otimes d_{Z}^{s}$. As $d_{T}^{s}$ is central in $\mathcal{O}_{q}\left(G L_{t}\right)$, we may apply Proposition 1.1(a) to conclude that $\gamma_{q}^{*}\left(\left(1 \otimes d_{Z}^{s}\right) w\right)=\gamma_{q}^{*}\left(1 \otimes d_{Z}^{s}\right) \gamma_{q}^{*}(w)=\left(d_{T}^{s} \otimes 1 \otimes d_{Z}^{s}\right)\left(d_{T}^{-s} \otimes w\right)=1 \otimes\left(\left(1 \otimes d_{Z}^{s}\right) w\right)$. obtain

(c) By Remark 3.2(i), $\rho_{q}^{*}\left(d_{Y}^{-s}\right)=d_{Y}^{-s} \otimes d_{T}^{-s}$. Since $S\left(d_{T}\right)=d_{T}^{-1}$, we thus 


$$
\gamma_{q}^{*}\left(d_{Y}^{-s} \otimes 1\right)=d_{T}^{s} \otimes d_{Y}^{-s} \otimes 1,
$$

and therefore part (c) follows from a second application of Proposition $1.1(\mathrm{a})$.

\section{§6. The First Fundamental Theorem for $\mathcal{O}_{q}\left(S L_{t}\right)$-coinvariants}

In this final section, we determine the $\Gamma_{q}^{*}$-coinvariants in $\mathcal{O}_{q}(V)$. Let $\mathscr{A}_{1}$ and $\mathscr{A}_{2}$ denote the respective subalgebras of $\mathcal{O}_{q}\left(M_{m, t}\right)$ and $\mathcal{O}_{q}\left(M_{t, n}\right)$ generated by all the $t \times t$ quantum minors. We shall prove that the set of $\Gamma_{q}^{*}$-coinvariants in $\mathcal{O}_{q}(V)$ is the subalgebra generated by $\mathscr{A}_{1} \otimes \mathscr{A}_{2}$ together with $\operatorname{Im} \mu_{q}^{*}$. In fact, this subalgebra turns out to be equal to the product $\left(\mathscr{A}_{1} \otimes \mathscr{A}_{2}\right) \cdot \operatorname{Im} \mu_{q}^{*}$. On the road to this goal, Theorem 5.5 puts us in a position roughly similar to that of Remark 4.1(ii), and to finish we need some computations analogous to Proposition 4.4 .

Until further notice, we continue to assume that $m=n>t$.

Remark 6.1. (i) As in Section 4, we set $\tilde{I}=\{n-t+1, \ldots, n\}$ and $\tilde{J}=$ $\{1, \ldots, t\}$. Let $P_{1}$ and $P_{2}$ denote the following ideals:

$$
\begin{aligned}
& \left.P_{1}=\langle\overline{[\tilde{I} \mid J]}| J \subseteq\{1, \ldots, n\} \text { and }|J|=t\right\rangle \triangleleft \mathcal{O}_{q}\left(M_{n}^{\leq t}\right) \\
& \left.P_{2}=\langle\overline{[I \mid \tilde{J}]}| I \subseteq\{1, \ldots, n\} \text { and }|I|=t\right\rangle \triangleleft \mathcal{O}_{q}\left(M_{n}^{\leq t}\right)
\end{aligned}
$$

These turn out to be completely prime ideals of $\mathcal{O}_{q}\left(M_{n}^{\leq t}\right)$ - see Remark 6.4. In view of $[2$, Corollary 5.2], the quantum minors $[\tilde{I} \mid J]$ can be arranged in a polynormal sequence, as can the $[I \mid \tilde{J}]$. Hence,

$$
P_{1}=\sum_{\substack{J \leq\{1, n\} \\|J|=t}} \overline{[\tilde{I} \mid J]} \mathcal{O}_{q}\left(M_{n}^{\leq t}\right) \quad \text { and } \quad P_{2}=\sum_{\substack{I \leq\{1, n\} \\|I|=t}} \overline{[I \mid \tilde{J}]} \mathcal{O}_{q}\left(M_{n}^{\leq t}\right)
$$

(ii) It can be proved that a $K$-basis for $P_{1}$ consists of all $\overline{\left[T \mid T^{\prime}\right]}$ where $\left(T, T^{\prime}\right)$ is a preferred bitableau with first row of the form $(\tilde{I}, ?)$, and that a $K$-basis for $P_{2}$ consists of all $\overline{\left[T \mid T^{\prime}\right]}$ where $\left(T, T^{\prime}\right)$ is a preferred bitableau with first row of the form $(?, \tilde{J})$. However, we shall not need these bases.

(iii) In view of point (i), powers of the $P_{i}$ can be expressed as follows:

$$
\begin{aligned}
& P_{1}^{s}=\sum_{\substack{J_{1},, J_{s} \leq\{1, n\} \\
\left|J_{1}\right|=\cdots=\left|J_{s}\right|=t}} \overline{\left[\tilde{I} \mid J_{1}\right]\left[\tilde{I} \mid J_{2}\right] \cdots\left[\tilde{I} \mid J_{s}\right]} \mathcal{O}_{q}\left(M_{n}^{\leq t}\right) \\
& P_{2}^{s}=\sum_{\substack{I_{1},, I_{s} \leq\{1, n, n\} \\
\left|I_{1}\right|=\cdots=\left|I_{s}\right|=t}} \overline{\left[I_{1} \mid \tilde{J}\right]\left[I_{2} \mid \tilde{J}\right] \cdots\left[I_{s} \mid \tilde{J}\right]} \mathcal{O}_{q}\left(M_{n}^{\leq t}\right)
\end{aligned}
$$

for $s=1,2, \ldots$. 
Lemma 6.2. Let $s$ be a nonnegative integer.

(a) A K-basis for $\left\langle d_{Y}^{s} \otimes 1\right\rangle$ consists of all pure tensors $\left[S \mid S^{\prime}\right] \otimes\left[T \mid T^{\prime}\right]$ in $\mathcal{O}_{q}(V)$ where $\left(S, S^{\prime}\right)$ and $\left(T, T^{\prime}\right)$ are preferred bitableaux and the first $s$ rows of $\left(S, S^{\prime}\right)$ equal $(\tilde{I}, \tilde{J})$.

(b) AK-basis for $\left\langle 1 \otimes d_{Z}^{s}\right\rangle$ consists of all pure tensors $\left[S \mid S^{\prime}\right] \otimes\left[T \mid T^{\prime}\right]$ in $\mathcal{O}_{q}(V)$ where $\left(S, S^{\prime}\right)$ and $\left(T, T^{\prime}\right)$ are preferred bitableaux and the first $s$ rows of $\left(T, T^{\prime}\right)$ equal $(\tilde{J}, \tilde{J})$.

Proof. Since $\left\langle d_{Y}^{s} \otimes 1\right\rangle=\left\langle d_{Y}^{s}\right\rangle \otimes \mathcal{O}_{q}\left(M_{t, n}\right)$ and $\left\langle 1 \otimes d_{Z}^{s}\right\rangle=\mathcal{O}_{q}\left(M_{n, t}\right) \otimes$ $\left\langle d_{Z}^{s}\right\rangle$, it suffices to check that the ideals $\left\langle d_{Y}^{s}\right\rangle$ and $\left\langle d_{Z}^{s}\right\rangle$ have bases of the appropriate forms. This can be done just as in Lemma 4.3(a)(b).

Proposition 6.3. If $s$ is any nonnegative integer, then

$$
\left(\mu_{q}^{*}\right)^{-1}\left(\left\langle d_{Y}^{s} \otimes 1\right\rangle\right)=P_{1}^{s} \quad \text { and } \quad\left(\mu_{q}^{*}\right)^{-1}\left(\left\langle 1 \otimes d_{Z}^{s}\right\rangle\right)=P_{2}^{s} .
$$

Proof. We prove the first equality; the proof of the second is analogous. The proof closely mimics that of Proposition 4.4.

In view of Remark 2.1(iii-v), we see that

$$
\mu_{q}^{*} \overline{[\tilde{I} \mid J]}=[\tilde{I} \mid \tilde{J}] \otimes[\tilde{J} \mid J]=d_{Y} \otimes[\tilde{J} \mid J]
$$

for all $t$-element subsets $J \subseteq\{1, \ldots, n\}$. Using Remark 6.1(iii) and the fact that $\mu_{q}^{*}$ is an algebra morphism, it follows that $P_{1}^{s} \subseteq\left(\mu_{q}^{*}\right)^{-1}\left(\left\langle d_{Y}^{s} \otimes 1\right\rangle\right)$.

If this inclusion is proper, choose an element

$$
x=\sum_{i=1}^{r} \alpha_{i} \overline{\left[T_{i} \mid T_{i}^{\prime}\right]} \in\left(\mu_{q}^{*}\right)^{-1}\left(\left\langle d_{Y}^{s} \otimes 1\right\rangle\right) \backslash P_{1}^{s}
$$

where the $\alpha_{i}$ are nonzero scalars, the $\left(T_{i}, T_{i}^{\prime}\right)$ are distinct preferred bitableaux, and the $T_{i}$ have at most $t$ columns. We may assume that none of the $\overline{\left[T_{i} \mid T_{i}^{\prime}\right]}$ lie in $P_{1}^{s}$. Let $\bar{\rho}$ be the minimum of the $\bar{\rho}\left(T_{i}\right)$ under reverse lexicographic order.

After re-indexing, we may assume that there is some $r^{\prime}$ such that $\bar{\rho}\left(T_{i}\right)=\bar{\rho}$ for $i \leq r^{\prime}$ and $\bar{\rho}\left(T_{i}\right)>_{\text {rlex }} \bar{\rho}$ for $i>r^{\prime}$. Applying [2, Lemma 2.3] to each $\mu_{q}^{*} \overline{\left[T_{i} \mid T_{i}^{\prime}\right]}$ and collecting terms, we see that

$$
\mu_{q}^{*}(x)=\sum_{i=1}^{r^{\prime}} \alpha_{i}\left[T_{i} \mid \mu\left(T_{i}\right)\right] \otimes\left[\mu^{\prime}\left(T_{i}\right) \mid T_{i}^{\prime}\right]+\sum_{j} X_{j} \otimes Y_{j}
$$

where the $X_{j}$ and $Y_{j}$ are homogeneous with $\bar{c}\left(X_{j}\right)=\bar{r}\left(Y_{j}\right)>_{\text {rlex }} \bar{\rho}$. We then observe that all of the $X_{j}$ belong to different homogeneous components than the $\left[T_{i} \mid \mu\left(T_{i}\right)\right]$ for $i \leq r^{\prime}$. Since

$$
\mu_{q}^{*}(x) \in\left\langle d_{Y}^{s} \otimes 1\right\rangle=\left\langle d_{Y}^{s}\right\rangle \otimes \mathcal{O}_{q}\left(M_{t, n}\right)
$$


and the ideal $\left\langle d_{Y}^{s}\right\rangle$ is homogeneous, it follows that

$$
\sum_{i=1}^{r^{\prime}} \alpha_{i}\left[T_{i} \mid \mu\left(T_{i}\right)\right] \otimes\left[\mu^{\prime}\left(T_{i}\right) \mid T_{i}^{\prime}\right] \in\left\langle d_{Y}^{s} \otimes 1\right\rangle .
$$

For $1 \leq i<j \leq r^{\prime}, \quad$ either $\quad\left(T_{i}, \mu\left(T_{i}\right)\right) \neq\left(T_{j}, \mu\left(T_{j}\right)\right) \quad$ or $\quad\left(\mu^{\prime}\left(T_{i}\right), T_{i}^{\prime}\right) \neq$ $\left(\mu^{\prime}\left(T_{j}\right), T_{j}^{\prime}\right)$. In view of Lemma 6.2(a), it follows that for $1 \leq i \leq r^{\prime}$, the first $s$ rows of $T_{i}$ equal $\tilde{I}$. But then $\overline{\left[T_{i} \mid T_{i}^{\prime}\right]} \in P_{1}^{s}$ for $1 \leq i \leq r^{\prime}$, which contradicts our choices above and therefore establishes the desired equality.

Remark 6.4. Although we shall not need the fact here, we note that the case $s=1$ of Proposition 6.3 implies that $P_{1}$ and $P_{2}$ are completely prime ideals of $\mathcal{O}_{q}\left(M_{n}^{\leq t}\right)$. To see this, it obviously suffices to show that $\left\langle d_{Y} \otimes 1\right\rangle$ and $\left\langle 1 \otimes d_{Z}\right\rangle$ are completely prime ideals of $\mathcal{O}_{q}(V)$. Observe that $\mathcal{O}_{q}\left(M_{n, t}\right) /\left\langle d_{Y}\right\rangle$ is an iterated skew polynomial ring over an isomorphic copy of $\mathcal{O}_{q}\left(M_{t}\right)$ modulo the ideal generated by its quantum determinant. Since the latter algebra is a domain ([3], [4, p. 182], or see [2, Theorem 2.5]), it follows that $\mathcal{O}_{q}\left(M_{n, t}\right) /\left\langle d_{Y}\right\rangle$ is a domain. Now the algebra

$$
\mathcal{O}_{q}(V) /\left\langle d_{Y} \otimes 1\right\rangle \cong\left(\mathcal{O}_{q}\left(M_{n, t}\right) /\left\langle d_{Y}\right\rangle\right) \otimes \mathcal{O}_{q}\left(M_{t, n}\right)
$$

is an iterated skew polynomial ring over $\mathcal{O}_{q}\left(M_{n, t}\right) /\left\langle d_{Y}\right\rangle$, and therefore the algebra $\mathcal{O}_{q}(V) /\left\langle d_{Y} \otimes 1\right\rangle$ is a domain. Thus $\left\langle d_{Y} \otimes 1\right\rangle$ is completely prime, and a similar argument shows that $\left\langle 1 \otimes d_{Z}\right\rangle$ is completely prime.

Lemma 6.5. Let $w \in \mathcal{O}_{q}(V)_{i, j}$ be a $\Gamma_{q}^{*}$-coinvariant.

(a) If $i \geq j$, then $w \in\left(\mathscr{A}_{1} \otimes 1\right) \cdot \operatorname{Im} \mu_{q}^{*}$.

(b) If $i \leq j$, then $w \in\left(1 \otimes \mathscr{A}_{2}\right) \cdot \operatorname{Im} \mu_{q}^{*}$.

Proof. By Theorem 5.5(a), the ratio $s=(i-j) / t$ is an integer.

(a) In this case, Theorem $5.5(\mathrm{~b})$ shows that $\left(1 \otimes d_{Z}^{s}\right) w$ is a $\gamma_{q}^{*}$-coinvariant. Thus, by Theorem 4.5, $\left(1 \otimes d_{Z}^{s}\right) w=\mu_{q}^{*}(x)$ for some $x \in \mathcal{O}_{q}\left(M_{n}^{\leq t}\right)$. Now $\mu_{q}^{*}(x) \in\left\langle 1 \otimes d_{Z}^{s}\right\rangle$, and hence $x \in P_{2}^{s}$, by Proposition 6.3. In view of Remark 6.1(iii), we thus have $x=\sum_{i=1}^{r} \bar{p}_{i} x_{i}$ for some $x_{i} \in \mathcal{O}_{q}\left(M_{n}^{\leq t}\right)$ and some $p_{i} \in \mathcal{O}_{q}\left(M_{n}\right)$ of the form

$$
p_{i}=\left[I_{i, 1} \mid \tilde{J}\right]\left[I_{i, 2} \mid \tilde{J}\right] \cdots\left[I_{i, s} \mid \tilde{J}\right] .
$$

Now by Remark 2.1(iii-v), each

$$
\mu_{q}^{*} \overline{\left[I_{i, l} \mid \tilde{J}\right]}=\left[I_{i, l} \mid \tilde{J}\right] \otimes[\tilde{J} \mid \tilde{J}]=\left[I_{i, l} \mid \tilde{J}\right] \otimes d_{Z}
$$

Since $\mu_{q}^{*}$ is an algebra morphism, it follows that each

$$
\mu_{q}^{*}\left(\bar{p}_{i} x_{i}\right)=\left(1 \otimes d_{Z}^{S}\right)\left(\left[I_{i, 1} \mid \tilde{J}\right] \otimes 1\right)\left(\left[I_{i, 2} \mid \tilde{J}\right] \otimes 1\right) \cdots\left(\left[I_{i, s} \mid \tilde{J}\right] \otimes 1\right) \mu_{q}^{*}\left(x_{i}\right) .
$$

Consequently, $\left(1 \otimes d_{Z}^{s}\right) w=\mu_{q}^{*}(x)=\left(1 \otimes d_{Z}^{s}\right) v$ for some $v \in\left(\mathscr{A}_{1} \otimes 1\right) \cdot \operatorname{Im} \mu_{q}^{*}$. 
Since $1 \otimes d_{Z}$ is a nonzero element of the domain $\mathcal{O}_{q}(V)$, we conclude that $w=v$, and the proof of part (a) is complete.

(b) This is proved in a similar fashion.

Theorem 6.6. Let $m, n, t$ be arbitrary positive integers, and let $\mathscr{A}_{1}$ and $\mathscr{A}_{2}$ denote the respective subalgebras of $\mathcal{O}_{q}\left(M_{m, t}\right)$ and $\mathcal{O}_{q}\left(M_{t, n}\right)$ generated by all the $t \times t$ quantum minors. The set of $\Gamma_{q}^{*}$-coinvariants in $\mathcal{O}_{q}(V)=\mathcal{O}_{q}\left(M_{m, t}\right) \otimes$ $\mathcal{O}_{q}\left(M_{t, n}\right)$ is the subalgebra generated by $\mathscr{A}_{1} \otimes \mathscr{A}_{2}$ and $\operatorname{Im} \mu_{q}^{*}$. More precisely,

$$
\left(\mathcal{O}_{q}\left(M_{m, t}\right) \otimes \mathcal{O}_{q}\left(M_{t, n}\right)\right)^{\operatorname{co} \mathcal{O}_{q}\left(S L_{t}\right)}=\left(\mathscr{A}_{1} \otimes \mathscr{A}_{2}\right) \cdot \mu_{q}^{*}\left(\mathcal{O}_{q}\left(M_{m, n}^{\leq t}\right)\right) .
$$

Proof. As in Remark 2.4, it is enough to consider the case that $m=n>t$; we leave the details of the reduction to the reader. Let $\mathscr{C}$ denote the set of $\Gamma_{q}^{*}$-coinvariants in $\mathcal{O}_{q}(V)$, and recall from Proposition 5.1 that $\mathscr{C}$ is a subalgebra of $\mathcal{O}_{q}(V)$ containing $\operatorname{Im} \mu_{q}^{*}$.

If $[I \mid J]$ is a $t \times t$ quantum minor in $\mathcal{O}_{q}\left(M_{n, t}\right)$ (necessarily, $J=\tilde{J}$ ), then as in Remark 2.1(iii)(iv), we see that $\rho_{q}^{*}[I \mid J]=[I \mid J] \otimes d_{T}$. It follows that $\gamma_{q}^{*}([I \mid J] \otimes 1)=d_{T}^{-1} \otimes[I \mid J] \otimes 1$, whence

$$
\Gamma_{q}^{*}([I \mid J] \otimes 1)=(\pi \otimes \mathrm{id})\left(d_{T}^{-1} \otimes[I \mid J] \otimes 1\right)=1 \otimes[I \mid J] \otimes 1
$$

and so $[I \mid J] \otimes 1 \in \mathscr{C}$. Similarly, $1 \otimes[I \mid J] \in \mathscr{C}$ for all $t \times t$ quantum minors $[I \mid J]$ in $\mathcal{O}_{q}\left(M_{t, n}\right)$. Therefore $\mathscr{C}$ contains the subalgebra of $\mathcal{O}_{q}(V)$ generated by $\mathscr{A}_{1} \otimes \mathscr{A}_{2}$ and $\operatorname{Im} \mu_{q}^{*}$.

On the other hand, it is immediate from Lemmas $5.2(\mathrm{~b})$ and 6.5 that $\mathscr{C}$ is contained in $\left(\mathscr{A}_{1} \otimes \mathscr{A}_{2}\right) \cdot \operatorname{Im} \mu_{q}^{*}$. The theorem follows.

\section{References}

[1] De Concini, C. and Procesi, C., A characteristic free approach to invariant theory, Adv. Math., 21 (1976), 330-354.

[2] Goodearl, K. R. and Lenagan, T. H., Quantum determinantal ideals, Duke Math. J. (to appear; available at http://www.math.ucsb.edu/ goodearl/preprints.html).

[ 3 ] Jordan, D. A., Notes (1993).

[4] Levasseur, T. and Stafford, J. T., The quantum coordinate ring of the special linear group, J. Pure Appl. Algebra, 86 (1993), 181-186.

[ 5] Montgomery, S., Hopf Algebras and their Actions on Rings, CBMS Regional Conf. Ser. 82, Providence (1993) Amer. Math. Soc.

[6] Noumi, M., Yamada, H. and Mimachi, K., Finite dimensional representations of the quantum group $G L_{q}(n ; \mathbf{C})$ and the zonal spherical functions on $U_{q}(n-1) \backslash U_{q}(n)$, Japan. J. Math., 19 (1993), 31-80.

[7] Parshall, B. and Wang, J.-P., Quantum linear groups, Mem. Amer. Math. Soc., 89 (1991), no. 439.

[ 8 ] Procesi, C., A Primer of Invariant Theory, Brandeis Lecture Notes 1, September 1982. 